\title{
The Hybrid Steepest Descent Method for Split Variational Inclusion and Constrained Convex Minimization Problems
}

\author{
Jitsupa Deepho and Poom Kumam \\ Department of Mathematics, Faculty of Science, King Mongkut's University of Technology Thonburi (KMUTT), \\ 126 Pracha Uthit Road, Bang Mod, Thung Khru, Bangkok 10140, Thailand
}

Correspondence should be addressed to Poom Kumam; poom.kum@kmutt.ac.th

Received 21 May 2014; Accepted 17 July 2014; Published 13 August 2014

Academic Editor: Vinay Kanwar

Copyright (C) 2014 J. Deepho and P. Kumam. This is an open access article distributed under the Creative Commons Attribution License, which permits unrestricted use, distribution, and reproduction in any medium, provided the original work is properly cited.

\begin{abstract}
We introduced an implicit and an explicit iteration method based on the hybrid steepest descent method for finding a common element of the set of solutions of a constrained convex minimization problem and the set of solutions of a split variational inclusion problem.
\end{abstract}

\section{Introduction}

Fixed-point optimization methods are very popular methods for solving the nonlinear problems such as variational inequality problems, optimization problems, inverse problems, and equilibrium problems. The convex feasibility problem (CFP) is used for modeling inverse problems which arise from phase retrieval problems and the intensity-modulated radiation therapy. Moreover, the well-known special case of CEP is a split feasibility problem (SFP).

Let $H_{1}$ and $H_{2}$ be two real Hilbert spaces with inner product $\langle$,$\rangle and norm \|\cdot\|$. Let $C$ and $Q$ be nonempty closed convex subsets of $H_{1}$ and $H_{2}$, respectively. Now, we recall that the split feasibility problem (SFP) is to find a point $x^{*}$ with the following property:

$$
x^{*} \in C, \quad A x^{*} \in Q,
$$

where $A \in B\left(H_{1}, H_{2}\right)$ and $B\left(H_{1}, H_{2}\right)$ denotes the family of all bounded linear operators $H_{1}$ to $H_{2}$. In 1994, the SFP was first introduced by Censor and Elfving [1], in finite-dimensional Hilbert spaces, for modeling inverse problems which arise from phase retrievals and in medical image reconstruction. A number of image reconstruction problems can be formulated as the SFP; see, for example, [2] and the references therein. Recently, it is found that the SFP can also be applied to study intensity modulated radiation therapy; see, for example, [3] and the references therein.
A special case of the SFP is following a convex constrained linear inverse problem [4] of finding an element $x^{*}$ such that

$$
x^{*} \in C, \quad A x^{*}=b .
$$

Recall that a mapping $S$ of $C$ is said to be a nonexpansive mapping such that

$$
\|S x-S y\| \leq\|x-y\|
$$

for all $x, y \in C$. Further, we consider the following fixed point problem (FPP) for a nonexpansive mapping $S: H_{1} \rightarrow H_{1}$. Find $x \in H_{1}$ such that

$$
S x=x .
$$

The solution set of FPP (4) is denoted by $F(S)$. It is well known that if $F(S) \neq \emptyset, F(S)$ is closed and convex. A mapping $T: H_{1} \rightarrow H_{1}$ is said to be an averaged mapping if it can be written as the average of an identity $I$ and a nonexpansive mapping $S$; that is,

$$
T=(1-\alpha) I+\alpha S,
$$

where $\alpha$ is a number in $(0,1)$. More precisely, we say that $T$ is $\alpha$-averaged. It is known that the projection is $(1 / 2)$-averaged. Consider the following constrained convex minimization problem:

$$
\operatorname{minimize}\{f(x): x \in C\} \text {, }
$$


where $C$ is a closed and convex subset of a Hilbert space $H_{1}$ and $f: C \rightarrow \mathbb{R}$ is a real valued convex function. If $f$ is Fréchet differentiable, then the gradient-projection algorithm (GPA) generates a sequence $\left\{x_{n}\right\}_{n=0}^{\infty}$ according to the following recursive formula:

$$
x_{n+1}=P_{C}\left(x_{n}-\gamma \nabla f\left(x_{n}\right)\right), \quad \forall n \geq 0,
$$

or more generally,

$$
x_{n+1}=P_{C}\left(x_{n}-\gamma_{n} \nabla f\left(x_{n}\right)\right), \quad \forall n \geq 0,
$$

where, in both (7) and (8), the initial guess $x_{0}$ is taken from $C$ arbitrarily and the parameters, $\gamma$ or $\gamma_{n}$, are positive real numbers satisfying certain conditions. The convergence of the algorithms (7) and (8) depends on the behavior of the gradient $\nabla f$. It is known that if $\nabla f$ is $\alpha$-strongly monotone and $L$-Lipschitzian with constants $\alpha, L \geq 0$, such that

$$
\begin{gathered}
\langle\nabla f(x)-\nabla f(y), x-y\rangle \geq \alpha\|x-y\|^{2}, \quad x, y \in C, \\
\|\nabla f(x)-\nabla f(y)\| \leq L\|x-y\|, \quad x, y \in C,
\end{gathered}
$$

then, for $0<\gamma<2 \alpha / L^{2}$, the operator

$$
T:=P_{C}(I-\gamma \nabla f)
$$

is a contraction; hence, the sequence $\left\{x_{n}\right\}_{n=0}^{\infty}$ defined by the GPA (7) converges in norm to the unique solution of (6). More generally, if the sequence $\left\{\gamma_{n}\right\}_{n=0}^{\infty}$ is chosen to satisfy the property

$$
0<\liminf _{n \rightarrow \infty} \gamma_{n} \leq \limsup _{n \rightarrow \infty} \gamma_{n}<\frac{2 \alpha}{L^{2}},
$$

then the sequence $\left\{x_{n}\right\}_{n=0}^{\infty}$ defined by the GPA (8) converges in norm to the unique minimizer of (6).

However, if the gradient $\nabla f$ fails to be strongly monotone, the operator $T$ defined in (10) may fail to be contractive; consequently, the sequence $\left\{x_{n}\right\}_{n=0}^{\infty}$ generated by the algorithm (7) may fail to converge strongly [5]. If $\nabla f$ is Lipschitzian, then the algorithms (7) and (8) can still converge in the weak topology under certain conditions $[6,7]$.

In 2011, Xu [5] gave an alternative operator-oriented approach to algorithm (8), namely, an averaged mapping approach. He gave his averaged mapping approach to the GPA (8) and the relaxed GPA. Moreover, he constructed an example which shows that the algorithm (7) does not converge in norm in an infinite-dimensional space and also presented two modifications of GPA which are shown to have strong convergence.

Given a mapping $A: C \rightarrow H_{1}$, the classical variational inequality problem (VIP) is to find $x^{*} \in C$ such that

$$
\left\langle A x^{*}, x-x^{*}\right\rangle \geq 0, \quad \forall x \in C .
$$

The solution set of VIP (12) is denoted by $\operatorname{VI}(C, A)$. It is well known that $x^{*} \in \mathrm{VI}(C, A)$ if and only if $x^{*}=P_{C}\left(x^{*}-\lambda A x^{*}\right)$ for some $\lambda>0$. The variational inequality was first discussed by Lions [8] and now is well known. The variational inequality theory has been studied quite extensively and has emerged as an important tool in the study of a wide class of obstacle, unilateral, free, moving, and equilibrium problems arising in several branches of pure and applied sciences in a unified and general framework.

Yamada [9] introduced the hybrid steepest descent method as follows:

$$
x_{n+1}=S x_{n}-\alpha_{n} \mu B S x_{n},
$$

where $x_{1}=x \in H_{1},\left\{\alpha_{n}\right\} \subset(0,1), B: H_{1} \rightarrow H_{1}$ is a strongly monotone and Lipschitz continuous mapping, and $\mu$ is a positive real number. He considered the variational inequality problem over the set of common fixed points of a finite family of nonexpansive mappings and proved strong convergence of the sequence generated by the method. Later, Tian [10] considered the following iterative method for a nonexpansive mapping $T: H_{1} \rightarrow H_{1}$ with $F(T) \neq \emptyset$ :

$$
x_{n+1}=\alpha_{n} \gamma f\left(x_{n}\right)+\left(I-\mu \alpha_{n} F\right) T x_{n}, \quad n \geq 1,
$$

where $F$ is a $k$-Lipschitzian and $\eta$-strongly monotone operator. He proved that the sequence $\left\{x_{n}\right\}$ generated by (14) converges to a fixed point $q \in F(T)$, which is the unique solution of the variational inequality

$$
\langle(\gamma f-\mu F) q, p-q\rangle \leq 0, \quad p \in F(T) .
$$

Recently, Moudafi [11] introduced the following split monotone variational inclusion problem (SMVIP). Find $x^{*} \in$ $H_{1}$ such that

$$
0 \in f_{1}\left(x^{*}\right)+B_{1}\left(x^{*}\right)
$$

and such that

$$
y^{*}=A x^{*} \in H_{2} \quad \text { solves } 0 \in f_{2}\left(y^{*}\right)+B_{2}\left(y^{*}\right) \text {, }
$$

where $B_{1}: H_{1} \rightarrow 2^{H_{1}}$ and $B_{2}: H_{2} \rightarrow 2^{H_{2}}$ are multivalued maximal monotone mappings.

Moudafi [11] introduced an iterative method for solving SMVIP (16)-(17), which can be seen an important generalization of an iterative method given by Censor et al. [12] for split variational inequality problem. As Moudafi noted in [11], SMVIP (16)-(17) includes a special case, the split common fixed point problem, split variational inequality problem, split zero problem, and split feasibility problem $[1,3,11,12]$ which have already been studied and used in practice as a model in intensity-modulated radiation therapy treatment planning; see $[1,3]$. This formalism is also at the core of modeling of many inverse problems arising for phase retrieval and other real-world problems, for instance, in sensor networks, in computerized tomography, and in data compression; see, for example, $[2,13]$.

If $f_{1} \equiv 0$ and $f_{2} \equiv 0$ then SMVIP (16)-(17) reduces to the following split variational inclusion problem (SVIP). Find $x^{*} \in H_{1}$ such that

$$
0 \in B_{1}\left(x^{*}\right)
$$

and such that

$$
y^{*}=A x^{*} \in H_{2} \quad \text { solves } 0 \in B_{2}\left(y^{*}\right) .
$$


When looked separately, (18) is the variational inclusion problem and we denoted its solution set by $\operatorname{SOLVIP}\left(B_{1}\right)$. The SVIP (18)-(19) constitutes a pair of variational inclusion problems which have to be solved so that the image $y^{*}=A x^{*}$ under a given bounded linear operator $A$ of the solution $x^{*}$ of SVIP (18) in $H_{1}$ is the solution of another SVIP (19) in another space $\mathrm{H}_{2}$; we denote the solution set of SVIP (19) by $\operatorname{SOLVIP}\left(B_{2}\right)$. The solution set of SVIP (18)-(19) is denoted by $\Gamma=\left\{x^{*} \in H_{1}: x^{*} \in \operatorname{SOLVIP}\left(B_{1}\right)\right.$ and $\left.A x^{*} \in \operatorname{SOLVIP}\left(B_{2}\right)\right\}$.

Very recently, Byrne et al. [14] studied the weak and strong convergence of the following iterative method for SVIP (18)(19): for given $x_{0} \in H_{1}$, compute the iterative sequence $\left\{x_{n}\right\}$ generated by the following iterative scheme:

$$
x_{n+1}=J_{\lambda}^{B_{1}}\left(x_{n}+\gamma A^{*}\left(J_{\lambda}^{B_{2}}-I\right) A x_{n}\right),
$$

for $\lambda>0$. In 2013, Kazmi and Rizvi [15] studied the strong convergence of the following iterative method:

$$
\begin{gathered}
u_{n}=J_{\lambda}^{B_{1}}\left(x_{n}+\gamma A^{*}\left(J_{\lambda}^{B_{2}}-I\right) A x_{n}\right) \\
x_{n+1}=\alpha_{n} f\left(x_{n}\right)+\left(1-\alpha_{n}\right) S u_{n},
\end{gathered}
$$

where $\lambda>0$ and $\gamma \in(0,1 / L), L$ is the spectral radius of the operator $A^{*} A$, and $A^{*}$ is the adjoint of $A$. He proved the sequence $\left\{x_{n}\right\}$ generated by (21) strongly convergence to fixed point of nonexpansive mapping $S$ and the solution set $\Gamma$ of SVIP (18)-(19).

In this paper, motivated by the work of Xu [5], Yamada [9], Tian [10], Byrne et al. [14], and Kazmi and Rizvi [15], we proved the strong convergence theorems for finding a common element of the set of solutions of a constrained convex minimization problem and the set of solutions of a split variational inclusion problem (18)-(19).

\section{Preliminaries}

Throughout this paper, we always write $\rightarrow$ for weak convergence and $\rightarrow$ for strong convergence. We need some facts and tools in a real Hilbert space $H_{1}$, which are listed below. For any $x \in H_{1}$, there exists a unique nearest point in $C$ denoted by $P_{C}(x)$ such that

$$
\left\|x-P_{C}(x)\right\| \leq\|x-y\|, \quad \forall y \in C .
$$

The mapping $P_{C}$ is called the metric projection of $H_{1}$ onto $C$. We know that $P_{C}$ is a nonexpansive mapping from $H_{1}$ onto $C$. It is also known that $P_{C}(x) \in C$ and satisfied

$$
\left\langle x-y, P_{C} x-P_{C} y\right\rangle \geq\left\|P_{C} x-P_{C} y\right\|^{2}, \quad \forall x, y \in H_{1} .
$$

Moreover, $P_{C} x$ is characterized by the fact that $P_{C} x \in C$ and

$$
\begin{gathered}
\left\langle x-P_{C}(x), y-P_{C}(x)\right\rangle \leq 0, \\
\|x-y\|^{2} \geq\left\|x-P_{C}(x)\right\|^{2}+\left\|y-P_{C}(x)\right\|^{2}, \\
\forall x \in H_{1}, y \in C .
\end{gathered}
$$

It is known that every nonexpansive operator $T: H_{1} \rightarrow$ $H_{1}$ satisfies, for all $(x, y) \in H_{1} \times H_{1}$, the inequality

$$
\begin{gathered}
\langle(x-T(x))-(y-T(y)), T(y)-T(x)\rangle \\
\leq \frac{1}{2}\|(T(x)-x)-(T(y)-y)\|^{2}
\end{gathered}
$$

and therefore, we get, for all $(x, y) \in H_{1} \times F(T)$,

$$
\langle x-T(x), y-T(x)\rangle \leq \frac{1}{2}\|T(x)-x\|^{2}
$$

(see, e.g., Theorem 3 in [16] and Theorem 1 in [17]).

Lemma 1. Let $H_{1}$ be a real Hilbert space. There hold the following identities:

(i) $\|x-y\|^{2}=\|x\|^{2}-\|y\|^{2}-2\langle x-y, y\rangle, \forall x, y \in H_{1}$;

(ii) $\|t x+(1-t) y\|^{2}=t\|x\|^{2}+(1-t)\|y\|^{2}-t(1-t)\|x-y\|^{2}$, $\forall t \in[0,1], \forall x, y \in H_{1}$.

Lemma 2 (see [7]). Assume that $\left\{\alpha_{n}\right\}$ is a sequence of nonnegative real numbers such that

$$
a_{n+1} \leq\left(1-\gamma_{n}\right) a_{n}+\delta_{n}
$$

where $\left\{\gamma_{n}\right\}$ is a sequence in $(0,1)$ and $\left\{\delta_{n}\right\}$ is a sequence such that

(i) $\sum_{n=1}^{\infty} \gamma_{n}=\infty$;

(ii) $\lim \sup _{n \rightarrow \infty}\left(\delta_{n} / \gamma_{n}\right) \leq 0$ or $\sum_{n=1}^{\infty}\left|\delta_{n}\right|<\infty$.

Then, $\lim _{n \rightarrow \infty} \alpha_{n}=0$.

Lemma 3 (see [18]). Let $B: H_{1} \rightarrow H_{1}$ be an L-Lipschitzian and $\eta$-strongly monotone operator on a Hilbert space $H_{1}$ with $L>0, \eta>0,0<\mu<2 \eta / L^{2}$, and $0<t<1$. Then $S=(I-$ $t \mu B): H_{1} \rightarrow H_{1}$ is a contraction with contractive coefficients $1-t \tau$ and $\tau=(1 / 2) \mu\left(2 \eta-\mu L^{2}\right)$.

Lemma 4. A nonlinear mapping $B$ whose domain is $D(B) \subseteq$ $H_{1}$ and range is $R(B) \subseteq H_{1}$ is said to be

(i) monotone, if

$$
\langle B x-B y, x-y\rangle \geq 0, \quad \forall x, y \in D(B) ;
$$

(ii) $\beta$-strongly monotone if there exists a constant $\beta>0$ such that

$$
\langle B x-B y, x-y\rangle \geq \beta\|x-y\|^{2}, \quad \forall x, y \in D(B) ;
$$

(iii) $v$-inverse strongly monotone (or, $v$-ism), if there exists a constant $v>0$ such that

$\langle B x-B y, x-y\rangle \geq v\|B x-B y\|^{2}, \quad \forall x, y \in D(B) ;$

(iv) firmly nonexpansive, if

$$
\langle B x-B y, x-y\rangle \geq\|B x-B y\|^{2}, \quad \forall x, y \in D(B) .
$$


A multivalued mapping $M: H_{1} \rightarrow 2^{H_{1}}$ is called monotone if, for all $x, y \in H_{1}, u \in M x$ and $v \in M y$ such that

$$
\langle x-y, u-v\rangle \geq 0 \text {. }
$$

A monotone mapping $M: H_{1} \rightarrow 2^{H_{1}}$ is maximal if $\operatorname{Graph}(M)$ is not properly contained in the graph of any other monotone mapping.

It is known that a monotone mapping $M$ is maximal if and only if, for $(x, u) \in H_{1} \times H_{1},\langle x-y, u-v\rangle \geq 0$, for every $(y, v) \in \operatorname{Graph}(M)$ implies that $u \in M x$.

Let $M: H_{1} \rightarrow 2^{H_{1}}$ be a multivalued maximal monotone mapping. Then, the resolvent mapping $J_{\lambda}^{M}: H_{1} \rightarrow H_{1}$ associated with $M$ is defined by

$$
J_{\lambda}^{M}(x):=(I+\lambda M)^{-1}(x), \quad \forall x \in H_{1},
$$

for some $\lambda>0$, where $I$ stands for identity operator on $H_{1}$.

We note that for all $\lambda>0$ the resolvent operator $J_{\lambda}^{M}$ is single-valued, nonexpansive, and firmly nonexpansive.

Lemma 5 (see [15]). SVIP (18)-(19) is equivalent to find $x^{*} \in$ $H_{1}$ such that $y^{*}=A x^{*} \in H_{2}$,

$$
x^{*}=J_{\lambda}^{B_{1}}\left(x^{*}\right), \quad y^{*}=J_{\lambda}^{B_{2}}\left(y^{*}\right), \quad \text { for some } \lambda>0 .
$$

Lemma 6 (see [19]). Let $V: C \rightarrow H_{1}$ be an l-Lipschitz mapping with coefficient $l \geq 0$ and $B: C \rightarrow H_{1}$ a strong positive bounded linear operator with $\bar{\gamma}>0$. Then for $0<\gamma<$ $(\mu \bar{\gamma} / l)$

$$
\begin{gathered}
\langle x-y,(\mu B-\gamma V) x-(\mu B-\gamma V) y\rangle \\
\geq(\mu \bar{\gamma}-\gamma l)\|x-y\|^{2}, \quad x, y \in H_{1} .
\end{gathered}
$$

This is, $\mu B-\gamma V$ is strongly monotone with coefficient $\mu \bar{\gamma}-\gamma l$.

Proposition 7 (see [20]). We have the following assertions.

(i) $T$ is nonexpansive if and only if the complement $I-T$ is $(1 / 2)$-ism.

(ii) If $T$ is $\nu$-ism and $\gamma>0$, then $\gamma T$ is $(\nu / \gamma)$-ism.

(iii) $T$ is averaged if and only if the complement $I-T$ is $\nu$-ism, for some $\nu>1 / 2$. Indeed, for $\alpha \in(0,1), T$ is $\alpha$-averaged if and only if $I-T$ is $(1 / 2 \alpha)$-ism.

Proposition 8 (see $[20,21]$ ). We have the following assertions.

(i) If $T=(1-\alpha) S+\alpha V$, for some $\alpha \in(0,1)$, $S$ is averaged and $V$ is nonexpansive, and then $T$ is averaged.

(ii) $T$ is firmly nonexpansive if and only if the complement $I-T$ is firmly nonexpansive.

(iii) If $T=(1-\alpha) S+\alpha V$, for some $\alpha \in(0,1)$, $S$ is firmly nonexpansive and $V$ is nonexpansive, and then $T$ is averaged.

(iv) The composite of finite many averaged mappings is averaged. That is, if each of the mappings $\left\{T_{i}\right\}_{i=1}^{N}$ is averaged, then so is the composite $T_{1} \circ T_{2} \circ \cdots \circ T_{N}$. In particular, if $T_{1}$ is $\alpha_{1}$-averaged and $T_{2}$ is $\alpha_{2}$-averaged, where $\alpha_{1}, \alpha_{2} \in(0,1)$, then the composite $T_{1} T_{2}$ is $\alpha$ averaged, where $\alpha=\alpha_{1}+\alpha_{2}-\alpha_{1} \alpha_{2}$.
Lemma 9 (see [18]). Let $H_{1}$ be a Hilbert space, $C$ a nonempty closed convex subset of $H_{1}$, and $T: C \rightarrow C$ a nonexpansive mapping with $F(T) \neq \emptyset$. If $\left\{x_{n}\right\}$ is a sequence in $C$ weakly converging to $x$ and if $\left\{(I-T) x_{n}\right\}$ converges strongly to $y$, then $(I-T) x=y$.

\section{Main Results}

Throughout the rest of this paper, we always assume that $V$ is an $l$-Lipschitzian mapping with coefficient $l \geq 0$, and $D$ is a strongly positive bounded linear operator with coefficient $\bar{\varsigma}$. Then we obtain that $D$ is $\|D\|$-Lipschitzian and $\bar{\varsigma}$-strongly monotone. Let $f: C \rightarrow \mathbb{R}$ be a real-valued convex function and assume that $\nabla f$ is an $L$-Lipschitzian mapping with $L \geq 0$.

Note that $\nabla f$ is $L$-Lipschitzian; it implies that $\nabla f$ is $(1 / L)$-ism, which then implies that $\gamma \nabla f$ is $(1 / \gamma L)$-ism. So by Proposition 7, its complement $I-\gamma \nabla f$ is $(\gamma L / 2)$-averaged. Since $P_{C}$ is $(1 / 2)$-averaged, we obtain from Proposition 8 that the composition $P_{C}(I-\gamma \nabla f)$ is $((2+\gamma L) / 4)$-averaged for $0<\gamma<2 / L$. Hence we have that, for each $n, P_{C}\left(I-\gamma_{n} \nabla f\right)$ is $\left(\left(2+\gamma_{n} L\right) / 4\right)$-averaged. Therefore, we can write

$$
P_{C}\left(I-\gamma_{n} \nabla f\right)=\frac{2-\gamma_{n} L}{4}+\frac{2+\gamma_{n} L}{4} S_{n}
$$

where $S_{n}$ is nonexpansive.

Suppose that minimization problem (6) is consistent and let $U$ denote its solution set. Assume that $0<\mu<2 \bar{\varsigma} /\|D\|^{2}$ and $0<\varsigma<\left(\mu\left(\bar{\varsigma}-\mu\|D\|^{2} / 2\right) / l\right)=\tau / l$.

Define a mapping $W_{n}=S_{n} J_{\lambda}^{B_{1}}\left(I+\xi A^{*}\left(J_{\lambda}^{B_{2}}-I\right) A\right)$. Since both $J_{\lambda}^{B_{1}}$ and $J_{\lambda}^{B_{2}}$ are firmly nonexpansive, they are averaged mappings. For $\xi \in(0,1 / L)$, the mapping $\left(I+\xi A^{*}\left(J_{\lambda}^{B_{2}}-\right.\right.$ I) $A$ ) is averaged. It follows from Proposition 8(iv) that the mapping $J_{\lambda}^{B_{1}}\left(I+\xi A^{*}\left(J_{\lambda}^{B_{2}}-I\right) A\right)$ is averaged and hence that is nonexpansive mapping. It is easy to see that $W_{n}$ is also nonexpansive mapping.

Consider the following mapping $G_{n}$ on $H_{1}$ defined by

$$
\begin{aligned}
G_{n} x= & \alpha_{n} \varsigma V\left(J_{\lambda}^{B_{1}}\left(x+\xi A^{*}\left(J_{\lambda}^{B_{2}}-I\right) A x\right)\right) \\
& +\left(I-\alpha_{n} \mu D\right) W_{n} x, \quad \forall x \in H_{1}, n \in \mathbb{N}
\end{aligned}
$$

where $\alpha_{n} \in(0,1)$. From Lemma 3, we have

$$
\begin{aligned}
& \left\|G_{n} x-G_{n} y\right\| \\
& =\| \alpha_{n} V\left(J_{\lambda}^{B_{1}}\left(x+\xi A^{*}\left(J_{\lambda}^{B_{2}}-I\right) A x\right)\right)+\left(I-\alpha_{n} \mu D\right) W_{n} x \\
& -\left[\alpha_{n} V\left(J_{\lambda}^{B_{1}}\left(y+\xi A^{*}\left(J_{\lambda}^{B_{2}}-I\right) A y\right)\right)\right. \\
& \left.+\left(I-\alpha_{n} \mu D\right) W_{n} y\right] \|
\end{aligned}
$$




$$
\begin{aligned}
\leq & \alpha_{n} \varsigma \| V\left(J_{\lambda}^{B_{1}}\left(I+\xi A^{*}\left(J_{\lambda}^{B_{2}}-I\right) A\right) x\right) \\
& \quad-V\left(J_{\lambda}^{B_{1}}\left(I+\xi A^{*}\left(J_{\lambda}^{B_{2}}-I\right) A\right) y\right) \| \\
& +\left(1-\alpha_{n} \tau\right)\left\|W_{n} x-W_{n} y\right\| \\
\leq & \alpha_{n} \varsigma\|V x-V y\|+\left(1-\alpha_{n} \tau\right)\left\|W_{n} x-W_{n} y\right\| \\
\leq & \alpha_{n} \varsigma l\|x-y\|+\left(1-\alpha_{n} \tau\right)\|x-y\| \\
= & \left(1-\alpha_{n}(\tau-\varsigma l)\right)\|x-y\| .
\end{aligned}
$$

Since $0<1-\alpha_{n}(\tau-\varsigma l)<1$, it follows that $G_{n}$ is a contraction. Therefore, by the Banach contraction principle, $G_{n}$ has a unique fixed point $x_{n}^{Z} \in H_{1}$ such that

$$
\begin{aligned}
x_{n}^{Z}= & \alpha_{n} \varsigma V\left(J_{\lambda}^{B_{1}}\left(x_{n}^{Z}+\xi\left(J_{\lambda}^{B_{2}}-I\right) A x_{n}^{Z}\right)\right) \\
& +\left(I-\alpha_{n} \mu D\right) W_{n} x_{n}^{Z} .
\end{aligned}
$$

For simplicity, we will write $x_{n}$ for $x_{n}^{Z}$, provided no confusion occurs. Next, we prove that the sequence $\left\{x_{n}\right\}$ converges strongly to a point $x^{*} \in \Omega:=U \cap \Gamma$ which solves the variational inequality

$$
\left\langle(\varsigma V-\mu D) x^{*}, p-x^{*}\right\rangle \leq 0, \quad \forall p \in \Omega .
$$

Equivalently, $x^{*}=P_{\Omega}(I-\mu D+\varsigma V) x^{*}$.

\subsection{An Implicit Iteration Method}

Theorem 10. Let $H_{1}$ and $H_{2}$ be two real Hilbert spaces and let $A: H_{1} \rightarrow H_{1}$ be a bounded linear operator, $f: C \rightarrow \mathbb{R} a$ real-value convex function, and $\nabla f$ an L-Lipschitzian mapping with $L \geq 0$. Assume that $\Omega:=U \cap \Gamma \neq \emptyset$. Let $V: C \rightarrow C$ be an l-Lipschitzain mapping with $l \geq 0$ and let $D$ be a strongly positive bounded linear operator with coefficients $\bar{\varsigma} \geq 0,0<$ $\mu<\left(2 \bar{\varsigma} /\|D\|^{2}\right)$, and $0<\varsigma<\left(\mu\left(\bar{\varsigma}-\left(\mu\|D\|^{2} / 2\right)\right) / l\right)=\tau / l$. Given $x_{0} \in H_{1}$ arbitrarily, let $\left\{x_{n}\right\}$ and $\left\{u_{n}\right\}$ be a sequence generated by the following algorithm:

$$
\begin{gathered}
u_{n}=J_{\lambda}^{B_{1}}\left(x_{n}+\xi A^{*}\left(J_{\lambda}^{B_{2}}-I\right) A x_{n}\right) \\
x_{n}=\alpha_{n} \varsigma V u_{n}+\left(I-\mu \alpha_{n} D\right) S_{n} u_{n},
\end{gathered}
$$

where $\xi \in(0,1 / L), P_{C}\left(I-\gamma_{n} \nabla f\right)=\beta_{n} I+\left(1-\beta_{n}\right) S_{n}, S_{n}$ is nonexpansive, $\beta_{n}=\left(2-\gamma_{n} L\right) / 4, \gamma_{n} \in(0,2 / L)$, and $A^{*}$ is the adjoint of $A$ and $\left\{\alpha_{n}\right\},\left\{\beta_{n}\right\}$ satisfying the following conditions:

(i) $\left\{\alpha_{n}\right\} \subset(0,1), \lim _{n \rightarrow \infty} \alpha_{n}=0$ and $\sum_{n=1}^{\infty}\left|\alpha_{n+1}-\alpha_{n}\right|<$ $\infty$;

(ii) $\left\{\beta_{n}\right\} \subset(0,1 / 2), \lim _{n \rightarrow \infty} \beta_{n}=0\left(\gamma_{n} \rightarrow 2 / L\right)$.

Then, the sequence $\left\{x_{n}\right\}$ converges strongly to a point $x^{*} \in \Omega$, which solves the variational inequality (40).

Proof. Consider the following.

Step 1. Show first that $\left\{x_{n}\right\}$ is bounded.
Since $\lim _{n \rightarrow \infty} \alpha_{n}=0$, we can assume that $\alpha_{n} \in\left(0,\|D\|^{-1}\right)$. By Lemma 3, we have $\left\|I-\alpha_{n} \mu D\right\| \leq 1-\alpha_{n} \tau$.

Let $p \in \Omega$; we have $p=J_{\lambda}^{B_{1}} p, A p=J_{\lambda}^{B_{2}}(A p)$. We estimate

$$
\begin{aligned}
\left\|u_{n}-p\right\|^{2} & \\
= & \left\|J_{\lambda}^{B_{1}}\left(x_{n}+\xi A^{*}\left(J_{\lambda}^{B_{2}}-I\right) A x_{n}\right)-p\right\|^{2} \\
= & \left\|J_{\lambda}^{B_{1}}\left(x_{n}+\xi A^{*}\left(J_{\lambda}^{B_{2}}-I\right) A x_{n}\right)-J_{\lambda}^{B_{1}} p\right\|^{2} \\
\leq & \left\|x_{n}+\xi A^{*}\left(J_{\lambda}^{B_{2}}-I\right) A x_{n}-p\right\|^{2} \\
\leq & \left\|x_{n}-p\right\|^{2}+\xi^{2}\left\|A^{*}\left(J_{\lambda}^{B_{2}}-I\right) A x_{n}\right\|^{2} \\
& +2 \xi\left\langle x_{n}-p, A^{*}\left(J_{\lambda}^{B_{2}}-I\right) A x_{n}\right\rangle .
\end{aligned}
$$

Thus, we have

$$
\begin{aligned}
\| u_{n}- & p \|^{2} \\
\leq & \left\|x_{n}-p\right\|^{2}+\xi^{2}\left\langle\left(J_{\lambda}^{B_{2}}-I\right) A x_{n}, A A^{*}\left(J_{\lambda}^{B_{2}}-I\right) A x_{n}\right\rangle \\
& +2 \xi\left\langle x_{n}-p, A^{*}\left(J_{\lambda}^{B_{2}}-I\right) A x_{n}\right\rangle .
\end{aligned}
$$

Now, we have

$$
\begin{aligned}
\xi^{2} & \left\langle\left(J_{\lambda}^{B_{2}}-I\right) A x_{n}, A A^{*}\left(J_{\lambda}^{B_{2}}-I\right) A x_{n}\right\rangle \\
& \leq L \xi^{2}\left\langle\left(J_{\lambda}^{B_{2}}-I\right) A x_{n},\left(J_{\lambda}^{B_{2}}-I\right) A x_{n}\right\rangle \\
& =L \xi^{2}\left\|\left(J_{\lambda}^{B_{2}}-I\right) A x_{n}\right\|^{2} .
\end{aligned}
$$

Setting $\Lambda:=2 \xi\left\langle x_{n}-p, A^{*}\left(J_{\lambda}^{B_{2}}-I\right) A x_{n}\right\rangle$ and using (26), we have

$$
\begin{aligned}
& \Lambda= 2 \xi\left\langle x_{n}-p, A^{*}\left(J_{\lambda}^{B_{2}}-I\right) A x_{n}\right\rangle \\
&= 2 \xi\left\langle A\left(x_{n}-p\right),\left(J_{\lambda}^{B_{2}}-I\right) A x_{n}\right\rangle \\
&= 2 \xi\left\langle A\left(x_{n}-p\right)+\left(J_{\lambda}^{B_{2}}-I\right) A x_{n}\right. \\
&\left.\quad-\left(J_{\lambda}^{B_{2}}-I\right) A x_{n},\left(J_{\lambda}^{B_{2}}-I\right) A x_{n}\right\rangle \\
&= 2 \xi\left\{\left\langle J_{\lambda}^{B_{2}} A x_{n}-A p,\left(J_{\lambda}^{B_{2}}-I\right) A x_{n}\right\rangle-\left\|\left(J_{\lambda}^{B_{2}}-I\right) A x_{n}\right\|^{2}\right\} \\
& \leq 2 \xi\left\{\frac{1}{2}\left\|\left(J_{\lambda}^{B_{2}}-I\right) A x_{n}\right\|^{2}-\left\|\left(J_{\lambda}^{B_{2}}-I\right) A x_{n}\right\|^{2}\right\} \\
& \leq-\xi\left\|\left(J_{\lambda}^{B_{2}}-I\right) A x_{n}\right\|^{2} .
\end{aligned}
$$

Using (43), (44), and (45), we obtain

$$
\left\|u_{n}-p\right\|^{2} \leq\left\|x_{n}-p\right\|^{2}+\xi(L \xi-1)\left\|\left(J_{\lambda}^{B_{2}}-I\right) A x_{n}\right\|^{2} .
$$

Since $\xi \in(0,1 / L)$, we obtain

$$
\left\|u_{n}-p\right\|^{2} \leq\left\|x_{n}-p\right\|^{2}
$$


Thus, by (41) and Lemma 3, we derive that

$$
\begin{aligned}
&\left\|x_{n}-p\right\| \\
&=\left\|\alpha_{n} \varsigma V u_{n}+\left(I-\mu \alpha_{n} D\right) S_{n} u_{n}-p\right\| \\
&=\|\left(I-\mu \alpha_{n} D\right) S_{n} u_{n}-\left(I-\mu \alpha_{n} D\right) S_{n} p \\
& \quad+\alpha_{n} \varsigma V u_{n}-\alpha_{n} \varsigma V p+\alpha_{n} \varsigma V p-\alpha_{n} \mu D p \| \\
& \leq\left(1-\alpha_{n} \tau\right)\left\|u_{n}-p\right\|+\alpha_{n} \varsigma l\left\|u_{n}-p\right\| \\
&+\alpha_{n}\|(\varsigma V-\mu D) p\| \\
& \leq\left(1-\alpha_{n}(\tau-\varsigma l)\right)\left\|x_{n}-p\right\|+\alpha_{n}\|(\varsigma V-\mu D) p\| \\
& \leq\left(1-\alpha_{n}(\tau-\varsigma l)\right)\left\|x_{n}-p\right\|+\alpha_{n}(\tau-\varsigma l) \frac{\|(\varsigma V-\mu D) p\|}{\tau-\varsigma l} .
\end{aligned}
$$

It follows that $\left\|x_{n}-p\right\| \leq\|(\varsigma V-\mu D) p\| /(\tau-\varsigma l)$.

Hence $\left\{x_{n}\right\}$ is bounded and so is $\left\{u_{n}\right\}$. It follows from the Lipschitz of $D, \nabla f, V$ that $\left\{D x_{n}\right\},\left\{D u_{n}\right\},\left\{\nabla f u_{n}\right\}$, and $\left\{V u_{n}\right\}$ are also bounded. From the nonexpansivity of $S_{n}$, it follows that $\left\{S_{n} u_{n}\right\}$ is also bounded.

Step 2. Show that

$$
\lim _{n \rightarrow \infty}\left\|x_{n}-u_{n}\right\|=0 .
$$

Next, from (46) and (47), we will show that

$$
\begin{aligned}
& \left\|x_{n}-p\right\|^{2} \\
& =\left\|\alpha_{n} \varsigma V u_{n}+\left(I-\mu \alpha_{n} D\right) S_{n} u_{n}-p\right\|^{2} \\
& =\|\left(I-\mu \alpha_{n} D\right) S_{n} u_{n}-\left(I-\mu \alpha_{n} D\right) S_{n} p \\
& +\alpha_{n} \varsigma V u_{n}-\alpha_{n} \mu D p \|^{2} \\
& \leq\left(1-\alpha_{n} \tau\right)^{2}\left\|u_{n}-p\right\|^{2}+2 \alpha_{n}\left\langle\varsigma V u_{n}-\mu D p, x_{n}-p\right\rangle \\
& =\left(1-\alpha_{n} \tau\right)^{2}\left\|u_{n}-p\right\|^{2} \\
& +2 \alpha_{n}\left\langle\varsigma V u_{n}-\varsigma V p+\varsigma V p-\mu D p, x_{n}-p\right\rangle \\
& =\left(1-\alpha_{n} \tau\right)^{2}\left\|u_{n}-p\right\|^{2}+2 \alpha_{n}\left\langle\varsigma V u_{n}-\varsigma V p, x_{n}-p\right\rangle \\
& +2 \alpha_{n}\left\langle\varsigma V p-\mu D p, x_{n}-p\right\rangle \\
& \leq\left(1-\alpha_{n} \tau\right)^{2}\left(\left\|x_{n}-p\right\|^{2}+\xi(L \xi-1)\left\|\left(J_{\lambda}^{B_{2}}-I\right) A x_{n}\right\|^{2}\right) \\
& +2 \alpha_{n} \iota l\left\|x_{n}-p\right\|^{2}+2 \alpha_{n}\|\varsigma V p-\mu D p\|\left\|x_{n}-p\right\| \\
& =\left(1-\alpha_{n} \tau\right)^{2}\left\|x_{n}-p\right\|^{2}-\left(1-\alpha_{n} \tau\right)^{2} \xi(1-L \xi) \\
& \times\left\|\left(J_{\lambda}^{B_{2}}-I\right) A x_{n}\right\|^{2}+2 \alpha_{n} s l\left\|x_{n}-p\right\|^{2} \\
& +2 \alpha_{n}\|\varsigma V p-\mu D p\|\left\|x_{n}-p\right\| .
\end{aligned}
$$

Therefore

$$
\begin{gathered}
\left(1-\alpha_{n} \tau\right)^{2} \xi(1-L \xi)\left\|\left(J_{\lambda}^{B_{2}}-I\right) A x_{n}\right\|^{2} \\
\leq\left(\alpha_{n} \tau\right)^{2}\left\|x_{n}-p\right\|^{2}+2 \alpha_{n} \varsigma l\left\|x_{n}-p\right\|^{2} \\
+2 \alpha_{n}\|\varsigma V p-\mu D p\|\left\|x_{n}-p\right\| .
\end{gathered}
$$

Since $\left(1-\alpha_{n} \tau\right)^{2} \xi(1-L \xi)>0$ and $\alpha_{n} \rightarrow 0$, we get

$$
\lim _{n \rightarrow \infty}\left\|\left(J_{\lambda}^{B_{2}}-I\right) A x_{n}\right\|=0 .
$$

Next, we estimate

$$
\begin{aligned}
\left\|u_{n}-p\right\|^{2} & \\
= & \left\|J_{\lambda}^{B_{1}}\left(x_{n}+\xi A^{*}\left(J_{\lambda}^{B_{2}}-I\right) A x_{n}\right)-p\right\|^{2} \\
= & \left\|J_{\lambda}^{B_{1}}\left(x_{n}+\xi A^{*}\left(J_{\lambda}^{B_{2}}-I\right) A x_{n}\right)-J_{\lambda}^{B_{1}} p\right\|^{2} \\
\leq & \left\langle u_{n}-p, x_{n}+\xi A^{*}\left(J_{\lambda}^{B_{2}}-I\right) A x_{n}-p\right\rangle \\
= & \frac{1}{2}\left\{\left\|u_{n}-p\right\|^{2}+\left\|x_{n}+\xi A^{*}\left(J_{\lambda}^{B_{2}}-I\right) A x_{n}-p\right\|^{2}\right. \\
& \left.\quad-\left\|\left(u_{n}-p\right)-\left[x_{n}+\xi A^{*}\left(J_{\lambda}^{B_{2}}-I\right) A x_{n}-p\right]\right\|^{2}\right\} \\
= & \frac{1}{2}\left\{\left\|u_{n}-p\right\|^{2}+\left\|x_{n}-p\right\|^{2}\right. \\
& +\xi(L \xi-1)\left\|\left(J_{\lambda}^{B_{2}}-I\right) A x_{n}-p\right\|^{2} \\
& \left.-\left\|u_{n}-x_{n}-\xi A^{*}\left(J_{\lambda}^{B_{2}}-I\right) A x_{n}\right\|^{2}\right\} \\
\leq & \frac{1}{2}\left\{\left\|u_{n}-p\right\|^{2}+\left\|x_{n}-p\right\|^{2}\right. \\
& \quad-\left[\left\|u_{n}-x_{n}\right\|^{2}+\xi^{2}\left\|A^{*}\left(J_{\lambda}^{B_{2}}-I\right) A x_{n}\right\|^{2}\right. \\
& \left.\left.-2 \xi\left\langle u_{n}-x_{n}, A^{*}\left(J_{\lambda}^{B_{2}}-I\right) A x_{n}\right\rangle\right]\right\} \\
\leq & \left.+2 \xi\left\|A\left(u_{n}-x_{n}\right)\right\|\left\|\left(J_{\lambda}^{B_{2}}-I\right) A x_{n}\right\|\right\} . \\
2 & \left\{u_{n}-p\left\|^{2}+\right\| x_{n}-p\left\|^{2}-\right\| u_{n}-x_{n} \|^{2}\right. \\
&
\end{aligned}
$$

So, we obtain

$$
\begin{aligned}
\left\|u_{n}-p\right\|^{2} \leq & \left\|x_{n}-p\right\|^{2}-\left\|u_{n}-x_{n}\right\|^{2} \\
& +2 \xi\left\|A\left(u_{n}-x_{n}\right)\right\|\left\|\left(J_{\lambda}^{B_{2}}-I\right) A x_{n}\right\| .
\end{aligned}
$$


Observe that, from (50) and (55), we get

$$
\begin{aligned}
\| x_{n}- & p \|^{2} \\
\leq & \left(1-\alpha_{n} \tau\right)^{2} \\
& \times\left(\left\|x_{n}-p\right\|^{2}-\left\|u_{n}-x_{n}\right\|^{2}\right. \\
& \left.+2 \xi\left\|A\left(u_{n}-x_{n}\right)\right\|\left\|\left(J_{\lambda}^{B_{2}}-I\right) A x_{n}\right\|\right) \\
& +2 \alpha_{n} \varsigma l\left\|x_{n}-p\right\|^{2}+2 \alpha_{n}\|\varsigma V p-\mu D p\|\left\|x_{n}-p\right\| \\
= & \left(1-\alpha_{n} \tau\right)^{2}\left\|x_{n}-p\right\|^{2}-\left(1-\alpha_{n} \tau\right)^{2}\left\|u_{n}-x_{n}\right\|^{2} \\
& +2\left(1-\alpha_{n} \tau\right)^{2} \xi\left\|A\left(u_{n}-x_{n}\right)\right\|\left\|\left(J_{\lambda}^{B_{2}}-I\right) A x_{n}\right\| \\
& +2 \alpha_{n} \varsigma l\left\|x_{n}-p\right\|^{2}+2 \alpha_{n}\|\varsigma V p-\mu D p\|\left\|x_{n}-p\right\| .
\end{aligned}
$$

Therefore

$$
\begin{aligned}
(1- & \left.\alpha_{n} \tau\right)^{2}\left\|u_{n}-x_{n}\right\|^{2} \\
\leq & \left(\alpha_{n} \tau\right)^{2}\left\|x_{n}-p\right\|^{2} \\
& +2\left(1-\alpha_{n} \tau\right)^{2} \xi\left\|A\left(u_{n}-x_{n}\right)\right\|\left\|\left(J_{\lambda}^{B_{2}}-I\right) A x_{n}\right\| \\
& +2 \alpha_{n} \varsigma l\left\|x_{n}-p\right\|^{2}+2 \alpha_{n}\|\varsigma V p-\mu D p\|\left\|x_{n}-p\right\| .
\end{aligned}
$$

Since $\left(1-\alpha_{n} \tau\right)^{2}>0, \alpha_{n} \rightarrow 0$, and as seen in (53), we get that (49) holds.

Step 3. Show that

$$
\lim _{n \rightarrow \infty}\left\|x_{n}-S_{n} x_{n}\right\|=0 .
$$

Observe that

$$
\begin{aligned}
& \left\|x_{n}-S_{n} x_{n}\right\| \\
& \quad=\left\|x_{n}-S_{n} u_{n}+S_{n} u_{n}-S_{n} x_{n}\right\| \\
& \quad \leq\left\|x_{n}-S_{n} u_{n}\right\|+\left\|S_{n} u_{n}-S_{n} x_{n}\right\| \\
& \quad \leq \alpha_{n}\left\|\varsigma V u_{n}-\mu D S_{n} u_{n}\right\|+\left\|u_{n}-x_{n}\right\| .
\end{aligned}
$$

Since $\alpha_{n} \rightarrow 0$ and as seen in (49), we get that (58) holds.

Thus,

$$
\begin{aligned}
& \left\|u_{n}-S_{n} u_{n}\right\| \\
& \quad=\left\|u_{n}-x_{n}+x_{n}-S_{n} x_{n}+S_{n} x_{n}-S_{n} u_{n}\right\| \\
& \quad \leq\left\|u_{n}-x_{n}\right\|+\left\|x_{n}-S_{n} x_{n}\right\|+\left\|S_{n} x_{n}-S_{n} u_{n}\right\| \\
& \quad \leq 2\left\|u_{n}-x_{n}\right\|+\left\|x_{n}-S_{n} x_{n}\right\| .
\end{aligned}
$$

From (49) and (58), we get $\lim _{n \rightarrow \infty}\left\|u_{n}-S_{n} u_{n}\right\|=0$.

Note that

$$
\begin{aligned}
& \left\|P_{C}\left(I-\gamma_{n} \nabla f\right) u_{n}-u_{n}\right\| \\
& \quad=\left\|\beta_{n} u_{n}+\left(1-\beta_{n}\right) S_{n} u_{n}-u_{n}\right\| \\
& =\left\|\left(1-\beta_{n}\right) S_{n} u_{n}-\left(1-\beta_{n}\right) u_{n}\right\| \\
& =\left(1-\beta_{n}\right)\left\|u_{n}-S_{n} u_{n}\right\| \leq\left\|u_{n}-S_{n} u_{n}\right\|,
\end{aligned}
$$

where $\beta_{n} \in(0,1 / 2)$. Since $\gamma_{n} \in(0,2 / L)$ and as seen in (61), we get

$$
\begin{aligned}
&\left\|P_{C}\left(I-\gamma_{n} \nabla f\right) u_{n}-u_{n}\right\| \\
&=\left\|P_{C}\left(I-\frac{2}{L} \nabla f\right) u_{n}-u_{n}\right\| \\
& \leq\left\|P_{C}\left(I-\frac{2}{L} \nabla f\right) u_{n}-P_{C}\left(I-\gamma_{n} \nabla f\right) u_{n}\right\| \\
& \quad+\left\|P_{C}\left(I-\gamma_{n} \nabla f\right) u_{n}-u_{n}\right\| \\
& \leq\left\|\left(I-\frac{2}{L} \nabla f\right) u_{n}-\left(I-\gamma_{n} \nabla f\right) u_{n}\right\| \\
& \quad+\left\|P_{C}\left(I-\gamma_{n} \nabla f\right) u_{n}-u_{n}\right\| \\
& \leq\left(\frac{2}{L}-\gamma_{n}\right)\left\|\nabla f\left(u_{n}\right)\right\|+\left\|S_{n} u_{n}-u_{n}\right\| .
\end{aligned}
$$

Since the boundedness of $\left\{\nabla f\left(u_{n}\right)\right\}, \beta_{n} \rightarrow 0$, and $\| S_{n} u_{n}-$ $u_{n} \| \rightarrow 0$, we conclude that

$$
\lim _{n \rightarrow \infty}\left\|P_{C}\left(I-\frac{2}{L} \nabla f\right) u_{n}-u_{n}\right\|=0
$$

So we conclude that

$$
\lim _{n \rightarrow \infty}\left\|P_{C}\left(I-\gamma_{n} \nabla f\right) u_{n}-u_{n}\right\|=0 \text {. }
$$

Since $\left\{u_{n}\right\}$ is bounded, there exists a subsequence $\left\{u_{n_{j}}\right\}$ which converges weakly to $x^{*}$.

Step 4. Show that $x^{*} \in \Omega$.

Since $C$ is closed and convex, $C$ is weakly closed so we have $x^{*} \in \Omega$. By Lemma 9 and (63), we have $x^{*} \in U$.

Next, show that $x^{*} \in \Gamma$.

Consider that $u_{n_{k}}=J_{\lambda}^{B_{1}}\left(x_{n_{k}}+\xi A^{*}\left(J_{\lambda}^{B_{2}}-I\right) A x_{n_{k}}\right)$ can be rewritten as

$$
\frac{\left(x_{n_{k}}-u_{n_{k}}\right)+A^{*}\left(J_{\lambda}^{B_{2}}-I\right) A x_{n_{k}}}{\lambda} \in B_{1} u_{n_{k}} .
$$

Taking limit $k \rightarrow \infty$ in (65) and by taking into account (49) and (53) and the fact that the graph of a maximal monotone operator is weakly strongly closed, we obtain $0 \in B_{1}\left(x^{*}\right)$; that is, $x^{*} \in \operatorname{SOLVIP}\left(B_{1}\right)$. Furthermore, since $\left\{x_{n}\right\}$ and $\left\{u_{n}\right\}$ have the same asymptotical behavior, $\left\{A x_{n_{k}}\right\}$ weakly converges to $A x^{*}$. Again, by (53) and the fact that the resolvent $J_{\lambda}^{B_{2}}$ is nonexpansive and Lemma 9, we obtain that $A x^{*} \in B_{2}\left(A x^{*}\right)$; that is, $A x^{*} \in \operatorname{SOLVIP}\left(B_{2}\right)$. Thus, $x^{*} \in \Omega$.

Step 5. Show that $x_{n} \rightarrow x^{*}$, where $x^{*}=P_{\Omega}(I-\mu D+\varsigma V) x^{*}$,

$$
\begin{aligned}
x_{n}-x^{*}= & \alpha_{n}\left(\varsigma V u_{n}-\mu D x^{*}\right)+\left(I-\mu \alpha_{n} D\right) S_{n} u_{n} \\
& -\left(I-\mu \alpha_{n} D\right) x^{*} .
\end{aligned}
$$


Hence, we obtain

$$
\begin{aligned}
\| x_{n}- & x^{*} \|^{2} \\
= & \alpha_{n}\left\langle\varsigma V u_{n}-\mu D x^{*}, x_{n}-x^{*}\right\rangle \\
& +\left\langle\left(I-\mu \alpha_{n} D\right) S_{n} u_{n}-\left(I-\mu \alpha_{n} D\right) x^{*}, x_{n}-x^{*}\right\rangle \\
\leq & \alpha_{n}\left\langle\varsigma V u_{n}-\mu D x^{*}, x_{n}-x^{*}\right\rangle+\left(1-\alpha_{n} \tau\right)\left\|x_{n}-x^{*}\right\|^{2} .
\end{aligned}
$$

It follows that

$$
\begin{aligned}
& \| x_{n}-x^{*} \|^{2} \\
& \leq \frac{1}{\tau}\left\langle\varsigma V u_{n}-\mu D x^{*}, x_{n}-x^{*}\right\rangle \\
&= \frac{1}{\tau}\left\langle\varsigma V u_{n}-\varsigma V x^{*}+\varsigma V x^{*}-\mu D x^{*}, x_{n}-x^{*}\right\rangle \\
&= \frac{1}{\tau}\left(\varsigma\left\langle V u_{n}-V x^{*}, x_{n}-x^{*}\right\rangle\right. \\
&\left.\quad \quad+\left\langle\varsigma V x^{*}-\mu D x^{*}, x_{n}-x^{*}\right\rangle\right) \\
& \leq \frac{1}{\tau}\left(\varsigma l\left\|x_{n}-x^{*}\right\|^{2}+\left\langle\varsigma V x^{*}-\mu D x^{*}, x_{n}-x^{*}\right\rangle\right) .
\end{aligned}
$$

This implies that

$$
\left\|x_{n}-x^{*}\right\|^{2} \leq \frac{\left\langle\varsigma V x^{*}-\mu D x^{*}, x_{n}-x^{*}\right\rangle}{\tau-\varsigma l} .
$$

In particular,

$$
\left\|x_{n_{j}}-x^{*}\right\| \leq \frac{\left\langle\varsigma V x^{*}-\mu D x^{*}, x_{n_{j}}-x^{*}\right\rangle}{\tau-\varsigma l} .
$$

Since $x_{n_{j}} \rightarrow x^{*}$, it follows from (70) that $x_{n_{j}} \rightarrow x^{*}$ as $j \rightarrow$ $\infty$.

Next, we show that $x^{*}$ solves the variational inequality (40). By the algorithm (41), we have

$$
x_{n}=\alpha_{n} \varsigma V u_{n}+\left(I-\mu \alpha_{n} D\right) S_{n} u_{n} .
$$

Therefore, we have

$$
\begin{aligned}
\alpha_{n}( & \mu D-\varsigma V) x_{n} \\
= & \alpha_{n}(\mu D-\varsigma V)\left(I-W_{n}\right) x_{n}+\alpha_{n}(\mu D-\varsigma V) S_{n} u_{n} \\
& +\alpha_{n} \varsigma V u_{n}+\left(I-\mu \alpha_{n} D\right) S_{n} u_{n}-x_{n} \\
= & \alpha_{n}(\mu D-\varsigma V)\left(I-W_{n}\right) x_{n}+\alpha_{n} \varsigma\left(V u_{n}-V S_{n} u_{n}\right) \\
& +S_{n} u_{n}-x_{n}
\end{aligned}
$$

$$
\begin{aligned}
= & \alpha_{n}(\mu D-\varsigma V)\left(I-W_{n}\right) x_{n}+\alpha_{n} \varsigma\left(V u_{n}-V S_{n} u_{n}\right) \\
& +S_{n} J_{\lambda}^{B_{1}}\left(x_{n}+\xi A^{*}\left(J_{\lambda}^{B_{2}}-I\right) A x_{n}\right)-x_{n} \\
= & \alpha_{n}(\mu D-\varsigma V)\left(I-W_{n}\right) x_{n}+\alpha_{n} \varsigma\left(V u_{n}-V S_{n} u_{n}\right) \\
& -\left[x_{n}-S_{n} J_{\lambda}^{B_{1}}\left(I+\xi A^{*}\left(J_{\lambda}^{B_{2}}-I\right) A\right) x_{n}\right] \\
= & \alpha_{n}(\mu D-\varsigma V)\left(I-W_{n}\right) x_{n}+\alpha_{n} \varsigma\left(V u_{n}-V S_{n} u_{n}\right) \\
& -\left(x_{n}-W_{n} x_{n}\right) \\
= & \alpha_{n}(\mu D-\varsigma V)\left(I-W_{n}\right) x_{n}+\alpha_{n} \varsigma\left(V u_{n}-V S_{n} u_{n}\right) \\
& -\left(I-W_{n}\right) x_{n} ;
\end{aligned}
$$

that is,

$$
\begin{aligned}
(\mu D-\varsigma V) x_{n}= & (\mu D-\varsigma V)\left(I-W_{n}\right) x_{n} \\
& +\varsigma\left(V u_{n}-V S_{n} u_{n}\right)-\frac{1}{\alpha_{n}}\left(I-W_{n}\right) x_{n} .
\end{aligned}
$$

Due to the nonexpansivity of $W_{n}$, we have that $I-W_{n}$ is monotone; that is, $\left\langle x-y,\left(I-W_{n}\right) x-\left(I-W_{n}\right) y\right\rangle \geq 0, \forall x, y \in$ $H_{1}$. Consider

$$
\begin{aligned}
\langle(\mu D & \left.-\varsigma V) x_{n}, x_{n}-p\right\rangle \\
= & -\frac{1}{\alpha_{n}}\left\langle\left(I-W_{n}\right) x_{n}-\left(I-W_{n}\right) p, x_{n}-p\right\rangle \\
& +\left\langle(\mu D-\varsigma V)\left(I-W_{n}\right) x_{n}, x_{n}-p\right\rangle \\
& +\varsigma\left\langle V u_{n}-V S_{n} u_{n}, x_{n}-p\right\rangle \\
\leq & \left\langle(\mu D-\varsigma V)\left(I-W_{n}\right) x_{n}, x_{n}-p\right\rangle \\
& +\varsigma\left\|V u_{n}-V S_{n} u_{n}\right\|\left\|x_{n}-p\right\| \\
\leq & \left\langle(\mu D-\varsigma V)\left(I-W_{n}\right) x_{n}, x_{n}-p\right\rangle \\
& +\varsigma l\left\|u_{n}-S_{n} u_{n}\right\|\left\|x_{n}-p\right\| .
\end{aligned}
$$

Now, by replacing $n$ in (74) with $n_{j}$ and taking $j \rightarrow \infty$, we get

$$
\begin{aligned}
& \left\langle(\mu D-\varsigma V) x^{*}, x^{*}-p\right\rangle \\
& \quad=\lim _{j \rightarrow \infty}\left\langle(\mu D-\varsigma V) x_{n_{j}}, x_{n_{j}}-p\right\rangle \\
& \quad \leq \lim _{j \rightarrow \infty}\left\langle(\mu D-\varsigma V)\left(x_{n_{j}}-S_{n_{j}} u_{n_{j}}\right), x_{n_{j}}-p\right\rangle=0 .
\end{aligned}
$$

That is, $x^{*} \in \Omega$ is a solution of the variational inequality (40).

Further, by the uniqueness of the solution of the variational inequality (40), we conclude that $x_{n} \rightarrow x^{*}$ as $n \rightarrow \infty$. We rewrite (40) as

$$
\left\langle(I-\mu D+\varsigma V) x^{*}-x^{*}, x^{*}-p\right\rangle \geq 0, \quad \forall p \in \Omega .
$$

This is equivalent to the fixed point equation

$$
P_{\Omega}(I-\mu D+\varsigma V) x^{*}=x^{*} .
$$




\subsection{An Explicit Iteration Method}

Theorem 11. Let $H_{1}$ and $H_{2}$ be two real Hilbert spaces and let $A: H_{1} \rightarrow H_{1}$ be a bounded linear operator, $f: C \rightarrow \mathbb{R} a$ real-value convex function, and $\nabla f$ an L-Lipschitzian mapping with $L \geq 0$. Assume that $\Omega:=U \cap \Gamma \neq \emptyset$. Let $V: C \rightarrow C$ be an l-Lipschitzain mapping with $l \geq 0$ and let $D$ be a strongly positive bounded linear operator with coefficients $\bar{\varsigma} \geq 0,0<$ $\mu<2 \bar{\varsigma} /\|D\|^{2}$, and $0<\varsigma<\mu\left(\bar{\varsigma}-\left(\mu\|D\|^{2} / 2\right)\right) / l=\tau / l$. Given $x_{0} \in H_{1}$ arbitrarily, let $\left\{x_{n}\right\}$ and $\left\{u_{n}\right\}$ be a sequence generated by the following algorithm:

$$
\begin{aligned}
& u_{n}=J_{\lambda}^{B_{1}}\left(x_{n}+\xi A^{*}\left(J_{\lambda}^{B_{2}}-I\right) A x_{n}\right) ; \\
& x_{n+1}=\alpha_{n} \varsigma V u_{n}+\left(I-\mu \alpha_{n} D\right) S_{n} u_{n},
\end{aligned}
$$

where $\xi \in(0,1 / L), P_{C}\left(I-\gamma_{n} \nabla f\right)=\beta_{n} I+\left(1-\beta_{n}\right) S_{n}, S_{n}$ is nonexpansive, $\beta_{n}=\left(2-\gamma_{n} L\right) / 4, \gamma_{n} \in(0,2 / L)$, and $A^{*}$ is the adjoint of $A$ and $\left\{\alpha_{n}\right\},\left\{\beta_{n}\right\}$ satisfying the following conditions:

(i) $\left\{\alpha_{n}\right\} \subset(0,1), \lim _{n \rightarrow \infty} \alpha_{n}=0$ and $\sum_{n=1}^{\infty}\left|\alpha_{n+1}-\alpha_{n}\right|<$ $\infty$;

(ii) $\left\{\beta_{n}\right\} \subset(0,1 / 2), \lim _{n \rightarrow \infty} \beta_{n}=0$ and $\sum_{n=1}^{\infty}\left|\beta_{n+1}-\beta_{n}\right|<$ $\infty$.

Then, the sequence $\left\{x_{n}\right\}$ converges strongly to a point $x^{*} \in \Omega$, which solves the variational inequality (40).

Proof. The proof is divided into several steps.

Step 1. Show first that $\left\{x_{n}\right\}$ is bounded.

Let $p \in \Omega$; we have $p=J_{\lambda}^{B_{1}} p$ and $A p=J_{\lambda}^{B_{2}}(A p)$. We have

$$
\left\|u_{n}-p\right\|^{2} \leq\left\|x_{n}-p\right\|^{2}
$$

Next, we derive that

$$
\begin{aligned}
\left\|x_{n+1}-p\right\| & \left\|\alpha_{n} \varsigma V u_{n}+\left(I-\mu \alpha_{n} D\right) S_{n} u_{n}-p\right\| \\
= & \|\left(I-\mu \alpha_{n} D\right) S_{n} u_{n}-\left(I-\mu \alpha_{n} D\right) S_{n} p \\
& \quad+\alpha_{n} \varsigma V u_{n}-\alpha_{n} \varsigma V p+\alpha_{n} \varsigma V p-\alpha_{n} \mu D p \| \\
\leq & \left(1-\alpha_{n} \tau\right)\left\|u_{n}-p\right\|+\alpha_{n} \varsigma l\left\|u_{n}-p\right\| \\
& +\alpha_{n}\|\varsigma V p-\mu D p\| \\
\leq & \left(1-\alpha_{n}(\tau-\varsigma l)\right)\left\|x_{n}-p\right\|+\alpha_{n}\|\varsigma V p-\mu B p\| \\
= & \left(1-\alpha_{n}(\tau-\varsigma l)\right)\left\|x_{n}-p\right\|+\alpha_{n}(\tau-\varsigma l) \frac{\|\varsigma V p-\mu D p\|}{\tau-\varsigma l} \\
\leq & \max \left\{\left\|x_{n}-p\right\|, \frac{\|\varsigma V p-\mu D p\|}{\tau-\varsigma l}\right\} .
\end{aligned}
$$

By induction, we obtain $\left\|x_{n}-p\right\| \leq \max \left\{\left\|x_{1}-p\right\|, \| \varsigma V p-\right.$ $\mu D p \| /(\tau-\varsigma l)\}, n \geq 1$. Hence, $\left\{x_{n}\right\}$ is bounded and so is $\left\{u_{n}\right\}$. It follows from the Lipschitz continuity of $D, \nabla f$, and
$V$ that $\left\{D x_{n}\right\},\left\{D u_{n}\right\},\left\{\nabla f\left(u_{n}\right)\right\}$, and $\left\{V u_{n}\right\}$ are also bounded. From the nonexpansivity of $S_{n}$, it follows that $\left\{S_{n} u_{n}\right\}$ is also bounded.

Step 2. Show that

$$
\left\|x_{n+1}-x_{n}\right\| \longrightarrow 0 \text { as } n \longrightarrow \infty \text {. }
$$

By (78), we have

$$
\begin{aligned}
\| x_{n+1}- & x_{n} \| \\
= & \| \alpha_{n} \varsigma V u_{n}+\left(I-\mu \alpha_{n} D\right) S_{n} u_{n}-\alpha_{n-1} \varsigma V u_{n-1} \\
& +\left(I-\mu \alpha_{n-1} D\right) S_{n-1} u_{n-1} \| \\
\leq & \alpha_{n} \varsigma\left\|V u_{n}-V u_{n-1}\right\|+\left|\alpha_{n}-\alpha_{n-1}\right| \varsigma\left\|V u_{n-1}\right\| \\
& +\left\|\left(I-\mu \alpha_{n} D\right) S_{n} u_{n}-\left(I-\mu \alpha_{n} D\right) S_{n} u_{n-1}\right\| \\
& +\left\|\left(I-\mu \alpha_{n} D\right) S_{n} u_{n-1}-\left(I-\mu \alpha_{n} D\right) S_{n-1} u_{n-1}\right\| \\
& +\left\|\left(I-\mu \alpha_{n} D\right) S_{n-1} u_{n-1}-\left(I-\mu \alpha_{n-1} D\right) S_{n-1} u_{n-1}\right\| \\
\leq & \alpha_{n} \varsigma l\left\|u_{n}-u_{n-1}\right\|+\left|\alpha_{n}-\alpha_{n-1}\right| \\
& \times\left(\left\|\varsigma V u_{n-1}\right\|+\left\|\mu D S_{n-1} u_{n-1}\right\|\right) \\
+ & \left(1-\alpha_{n} \tau\right)\left(\left\|u_{n}-u_{n-1}\right\|+\left\|S_{n} u_{n-1}-S_{n-1} u_{n-1}\right\|\right) \\
\leq & \left(1-\alpha_{n}(\tau-\varsigma l)\right)\left\|u_{n}-u_{n-1}\right\| \\
& +\left|\alpha_{n}-\alpha_{n-1}\right|\left(\left\|\varsigma V u_{n-1}\right\|+\left\|\mu D S_{n-1} u_{n-1}\right\|\right) \\
& +\left(1-\alpha_{n} \tau\right)\left\|S_{n} u_{n-1}-S_{n-1} u_{n-1}\right\| .
\end{aligned}
$$

Next, we estimate $\left\|S_{n} u_{n-1}-S_{n-1} u_{n-1}\right\|$. Observe that

$$
\begin{aligned}
\left\|S_{n} u_{n-1}-S_{n-1} u_{n-1}\right\| & \| \frac{P_{C}\left(I-\gamma_{n} \nabla f\right)-\beta_{n} I}{1-\beta_{n}} u_{n-1} \\
& -\frac{P_{C}\left(I-\gamma_{n-1} \nabla f\right)-\beta_{n-1} I}{1-\beta_{n-1}} u_{n-1} \| \\
= & \| \frac{4 P_{C}\left(I-\gamma_{n} \nabla f\right)-\left(2-\lambda_{n} L\right) I}{2+\gamma_{n} L} u_{n-1} \\
& -\frac{4 P_{C}\left(I-\gamma_{n-1} \nabla f\right)-\left(2-\gamma_{n-1} L\right) I}{2+\gamma_{n-1} L} u_{n-1} \| \\
\leq & \left\|\frac{4 P_{C}\left(I-\gamma_{n} \nabla f\right)}{2+\lambda_{n} L} u_{n-1}-\frac{4 P_{C}\left(I-\gamma_{n-1} \nabla f\right)}{2+\gamma_{n-1} L} u_{n-1}\right\| \\
& +\left\|\frac{2-\gamma_{n-1} L}{2+\gamma_{n-1} L} u_{n-1}-\frac{2-\gamma_{n} L}{2+\gamma_{n} L} u_{n-1}\right\|
\end{aligned}
$$




$$
\begin{aligned}
& =\|\left(4\left(2+\gamma_{n-1} L\right) P_{C}\left(I-\gamma_{n} \nabla f\right) u_{n-1}\right. \\
& \left.-4\left(2+\gamma_{n} L\right) P_{C}\left(I-\gamma_{n-1} \nabla f\right) u_{n-1}\right) \\
& \times\left(\left(2+\gamma_{n} L\right)\left(2+\lambda_{n-1} L\right)\right)^{-1} \| \\
& +\frac{4 L\left|\gamma_{n}-\gamma_{n-1}\right|}{\left(2+\gamma_{n} L\right)\left(2+\gamma_{n-1} L\right)}\left\|u_{n-1}\right\| \\
& \leq \| \frac{4 L\left(\gamma_{n-1}-\gamma_{n}\right) P_{C}\left(I-\gamma_{n} \nabla f\right) u_{n-1}}{\left(2+\gamma_{n} L\right)\left(2+\gamma_{n-1} L\right)} \\
& +\left(4 ( 2 + \gamma _ { n } L ) \left(P_{C}\left(I-\gamma_{n} \nabla f\right) u_{n-1}\right.\right. \\
& \left.\left.-P_{C}\left(I-\gamma_{n-1} \nabla f\right) u_{n-1}\right)\right) \\
& \times\left(\left(2+\lambda_{n} L\right)\left(2+\gamma_{n-1} L\right)\right)^{-1} \\
& +\frac{4 L\left|\gamma_{n}-\gamma_{n-1}\right|}{\left(2+\gamma_{n} L\right)\left(2+\gamma_{n-1} L\right)}\left\|u_{n-1}\right\| \\
& \leq \frac{4 L\left|\gamma_{n}-\gamma_{n-1}\right|\left\|P_{C}\left(I-\gamma_{n} \nabla f\right) u_{n-1}\right\|}{\left(2+\gamma_{n} L\right)\left(2+\gamma_{n-1} L\right)} \\
& +\frac{4\left\|P_{C}\left(I-\gamma_{n} \nabla f\right) u_{n-1}-P_{C}\left(I-\gamma_{n-1} \nabla f\right) u_{n-1}\right\|}{2+\gamma_{n-1} L} \\
& +\frac{4 L\left|\gamma_{n}-\gamma_{n-1}\right|}{\left(2+\gamma_{n} L\right)\left(2+\gamma_{n-1} L\right)}\left\|u_{n-1}\right\| \\
& \leq\left|\gamma_{n}-\gamma_{n-1}\right| \\
& \times\left(L\left\|P_{C}\left(I-\gamma_{n} \nabla f\right) u_{n-1}\right\|+2\left\|\nabla f\left(u_{n-1}\right)\right\|+L\left\|u_{n-1}\right\|\right) \\
& =\frac{4}{L}\left|\beta_{n}-\beta_{n-1}\right| \\
& \times\left(L\left\|P_{C}\left(I-\gamma_{n} \nabla f\right) u_{n-1}\right\|+2\left\|\nabla f\left(u_{n-1}\right)\right\|+L\left\|u_{n-1}\right\|\right) \\
& \leq M_{1}\left|\beta_{n}-\beta_{n-1}\right| \text {, }
\end{aligned}
$$

where $M_{1}=\sup _{n}\left\{4\left\|P_{C}\left(I-\gamma_{n} \nabla f\right) u_{n-1}\right\|+(8 / L)\left\|\nabla f\left(u_{n-1}\right)\right\|+\right.$ $\left.4\left\|u_{n-1}\right\|\right\}$.

Substitute (83) into (82); we get

$$
\begin{aligned}
\| x_{n+1} & -x_{n} \| \\
\leq & \left(1-\alpha_{n}(\tau-\varsigma l)\right)\left\|u_{n}-u_{n-1}\right\| \\
& +\left|\alpha_{n}-\alpha_{n-1}\right|\left(\left\|\varsigma V u_{n-1}\right\|+\left\|\mu D S_{n-1} u_{n-1}\right\|\right) \\
& +M_{1}\left|\beta_{n}-\beta_{n-1}\right| \\
\leq & \left(1-\alpha_{n}(\tau-\varsigma l)\right)\left\|u_{n}-u_{n-1}\right\| \\
& +\left(\left|\alpha_{n}-\alpha_{n-1}\right|+\left|\beta_{n}-\beta_{n-1}\right|\right) \\
& \times\left(\left\|\varsigma V u_{n-1}\right\|+\left\|\mu D S_{n-1} u_{n-1}\right\|+M_{1}\right)
\end{aligned}
$$

$$
\begin{aligned}
\leq & \left(1-\alpha_{n}(\tau-\varsigma l)\right)\left\|u_{n}-u_{n-1}\right\| \\
& +M_{2}\left(\left|\alpha_{n}-\alpha_{n-1}\right|+\left|\beta_{n}-\beta_{n-1}\right|\right),
\end{aligned}
$$

for some approximate positive constant $M_{2}$ such that

$$
M_{2}=\sup _{n}\left\{\left\|\varsigma V u_{n-1}\right\|+\left\|\mu D S_{n-1} u_{n-1}\right\|+M_{1}\right\} .
$$

Since, for $\xi \in(0,1 / L)$, the mapping $J_{\lambda}^{B_{1}}\left(I+\xi A^{*}\left(J_{\lambda}^{B_{2}}-I\right) A\right)$ is averaged and hence nonexpansive, we obtain

$$
\begin{aligned}
& \left\|u_{n}-u_{n-1}\right\| \\
& \leq \| J_{\lambda}^{B_{1}}\left(x_{n}+\xi A^{*}\left(J_{\lambda}^{B_{2}}-I\right) A x_{n}\right) \\
& \quad-J_{\lambda}^{B_{1}}\left(x_{n-1}+\xi A^{*}\left(J_{\lambda}^{B_{2}}-I\right) A x_{n-1}\right) \| \\
& \leq \| J_{\lambda}^{B_{1}}\left(I+\xi A^{*}\left(J_{\lambda}^{B_{2}}-I\right) A\right) x_{n} \\
& \quad-J_{\lambda}^{B_{1}}\left(I+\xi A^{*}\left(J_{\lambda}^{B_{2}}-I\right) A\right) x_{n-1} \| \\
& \leq\left\|x_{n}-x_{n-1}\right\| .
\end{aligned}
$$

Substitute (86) into (84); we get

$$
\begin{aligned}
\left\|x_{n+1}-x_{n}\right\| \leq & \left(1-\alpha_{n}(\tau-\varsigma l)\right)\left\|x_{n}-x_{n-1}\right\| \\
& +M_{2}\left(\left|\alpha_{n}-\alpha_{n-1}\right|+\left|\beta_{n}-\beta_{n-1}\right|\right) .
\end{aligned}
$$

By Lemma 2, it follows from conditions (i) to (ii) that (81) holds. Further, from (86), this implies that

$$
\left\|u_{n+1}-u_{n}\right\| \longrightarrow 0 \quad \text { as } n \longrightarrow \infty
$$

Step 3. Show that

$$
\left\|x_{n}-S_{n} x_{n}\right\| \longrightarrow 0 \text { as } n \longrightarrow \infty \text {. }
$$

From (55) and (78), we have

$$
\begin{aligned}
&\left\|x_{n+1}-p\right\|^{2} \\
&=\left\|\alpha_{n} \varsigma V u_{n}+\left(I-\mu \alpha_{n} D\right) S_{n} u_{n}-p\right\|^{2} \\
&=\|\left(I-\mu \alpha_{n} D\right) S_{n} u_{n}-\left(I-\mu \alpha_{n} D\right) S_{n} p \\
& \quad+\alpha_{n} \varsigma V u_{n}-\alpha_{n} \mu D p \|^{2} \\
& \leq\left(1-\alpha_{n} \tau\right)^{2}\left\|u_{n}-p\right\|^{2} \\
&+2\left(1-\alpha_{n} \tau\right) \alpha_{n}\left\|u_{n}-p\right\|\left\|\varsigma V u_{n}-\mu D p\right\| \\
&+\alpha_{n}^{2}\left\|\varsigma V u_{n}-\mu D p\right\|^{2}
\end{aligned}
$$




$$
\begin{aligned}
\leq & \left(1-\alpha_{n} \tau\right)^{2}\left(\left\|x_{n}-p\right\|^{2}-\left\|u_{n}-x_{n}\right\|^{2}\right. \\
& \left.+2 \xi\left\|A\left(u_{n}-x_{n}\right)\right\|\left\|\left(J_{\lambda}^{B_{2}}-I\right) A x_{n}\right\|\right) \\
& +2\left(1-\alpha_{n} \tau\right) \alpha_{n}\left\|u_{n}-p\right\|\left\|\varsigma V u_{n}-\mu D p\right\| \\
& +\alpha_{n}^{2}\left\|\varsigma V u_{n}-\mu D p\right\|^{2} \\
= & \left(1-\alpha_{n} \tau\right)^{2}\left\|x_{n}-p\right\|^{2}-\left(1-\alpha_{n} \tau\right)^{2}\left\|u_{n}-x_{n}\right\|^{2} \\
& +2\left(1-\alpha_{n} \tau\right)^{2} \xi\left\|A\left(u_{n}-x_{n}\right)\right\|\left\|\left(J_{\lambda}^{B_{2}}-I\right) A x_{n}\right\| \\
& +2\left(1-\alpha_{n} \tau\right) \alpha_{n}\left\|u_{n}-p\right\|\left\|\varsigma V u_{n}-\mu D p\right\| \\
& +\alpha_{n}^{2}\left\|\varsigma V u_{n}-\mu D p\right\|^{2} .
\end{aligned}
$$

This implies that

$$
\begin{aligned}
(1- & \left.\alpha_{n} \tau\right)^{2}\left\|u_{n}-x_{n}\right\|^{2} \\
\leq & \left\|x_{n}-p\right\|^{2}+\left(\alpha_{n} \tau\right)^{2}\left\|x_{n}-p\right\|^{2}-\left\|x_{n+1}-p\right\|^{2} \\
& +2\left(1-\alpha_{n} \tau\right)^{2} \xi\left\|A\left(u_{n}-x_{n}\right)\right\|\left\|\left(J_{\lambda}^{B_{2}}-I\right) A x_{n}\right\| \\
& +2\left(1-\alpha_{n} \tau\right) \alpha_{n}\left\|u_{n}-p\right\|\left\|\varsigma V u_{n}-\mu D p\right\| \\
& +\alpha_{n}^{2}\left\|\varsigma V u_{n}-\mu D p\right\|^{2} \\
\leq & \left(\left\|x_{n}-p\right\|+\left\|x_{n+1}-p\right\|\right)\left\|x_{n}-x_{n+1}\right\| \\
& +\left(\alpha_{n} \tau\right)^{2}\left\|x_{n}-p\right\|^{2} \\
& +2\left(1-\alpha_{n} \tau\right)^{2} \xi\left\|A\left(u_{n}-x_{n}\right)\right\|\left\|\left(J_{\lambda}^{B_{2}}-I\right) A x_{n}\right\| \\
& +2\left(1-\alpha_{n} \tau\right) \alpha_{n}\left\|u_{n}-p\right\|\left\|\varsigma V u_{n}-\mu D p\right\| \\
& +\alpha_{n}^{2}\left\|\varsigma V u_{n}-\mu D p\right\|^{2} .
\end{aligned}
$$

Since $\left(1-\alpha_{n} \tau\right)^{2}>0, \alpha_{n} \rightarrow 0$, and as seen in (53) and in (81), we get

$$
\lim _{n \rightarrow \infty}\left\|u_{n}-x_{n}\right\|=0
$$

Next,

$$
\begin{aligned}
\| x_{n}- & S_{n} x_{n} \| \\
= & \left\|x_{n}-x_{n+1}+x_{n+1}-S_{n} u_{n}+S_{n} u_{n}-S_{n} x_{n}\right\| \\
\leq & \left\|x_{n}-x_{n+1}\right\|+\left\|x_{n+1}-S_{n} u_{n}\right\| \\
& +\left\|S_{n} u_{n}-S_{n} x_{n}\right\| \\
\leq & \left\|x_{n}-x_{n+1}\right\|+\alpha_{n}\left\|\varsigma V u_{n}-\mu D S_{n} u_{n}\right\| \\
& +\left\|u_{n}-x_{n}\right\| .
\end{aligned}
$$

It follows from condition $\alpha_{n} \rightarrow 0$, (81), and (92) that (89) holds. Furthermore we have

$$
\begin{aligned}
& \left\|u_{n}-S_{n} u_{n}\right\| \\
& \quad=\left\|u_{n}-x_{n}+x_{n}-S_{n} x_{n}+S_{n} x_{n}-S_{n} u_{n}\right\| \\
& \quad \leq\left\|u_{n}-x_{n}\right\|+\left\|x_{n}-S_{n} x_{n}\right\|+\left\|x_{n}-u_{n}\right\| \\
& \quad \leq 2\left\|u_{n}-x_{n}\right\|+\left\|x_{n}-S_{n} x_{n}\right\| .
\end{aligned}
$$

It follows from (89) and (92) that $\left\|u_{n}-S_{n} u_{n}\right\| \rightarrow 0$.

Step 4. Show that

$$
\limsup _{n \rightarrow \infty}\left\langle(\varsigma V-\mu D) x^{*}, x_{n}-x^{*}\right\rangle \leq 0
$$

where $x^{*}=P_{\Omega}(I-\mu D+\varsigma V) x^{*}$ is a unique solution of the variational inequality (40). Indeed, take a subsequence $\left\{x_{n_{j}}\right\}$ of $\left\{x_{n}\right\}$ such that

$$
\begin{aligned}
& \limsup _{n \rightarrow \infty}\left\langle(\varsigma V-\mu D) x^{*}, x_{n}-x^{*}\right\rangle \\
& \quad=\lim _{j \rightarrow \infty}\left\langle(\varsigma V-\mu D) x^{*}, x_{n_{j}}-x^{*}\right\rangle .
\end{aligned}
$$

Since $\left\{x_{n_{j}}\right\}$ is bounded, there exists a subsequence $\left\{x_{n_{j_{k}}}\right\}$ of $\left\{x_{n_{j}}\right\}$ which converges weakly to $q$. Without loss of generality, we can assume that $x_{n_{j}} \rightarrow q$. Since $x^{*}=P_{\Omega}(I-\mu D+\varsigma V) x^{*}$, it follows that

$$
\begin{aligned}
& \limsup _{n \rightarrow \infty}\left\langle(\varsigma V-\mu D) x^{*}, x_{n}-x^{*}\right\rangle \\
& \quad=\lim _{j \rightarrow \infty}\left\langle(\varsigma V-\mu D) x^{*}, x_{n_{j}}-x^{*}\right\rangle \\
& =\left\langle(\varsigma V-\mu D) x^{*}, q-x^{*}\right\rangle \leq 0 .
\end{aligned}
$$

This implies that (95) holds.

Step 5. Show that

$$
x_{n} \longrightarrow x^{*} \text {, }
$$

since

$$
\begin{aligned}
\langle(\varsigma V & \left.-\mu D) x^{*}, x_{n+1}-x^{*}\right\rangle \\
= & \left\langle(\varsigma V-\mu D) x^{*}, x_{n+1}-x_{n}+x_{n}-x^{*}\right\rangle \\
= & \left\langle(\varsigma V-\mu D) x^{*}, x_{n+1}-x_{n}\right\rangle \\
& +\left\langle(\varsigma V-\mu D) x^{*}, x_{n}-x^{*}\right\rangle \\
\leq & \left\|(\varsigma V-\mu D) x^{*}\right\|\left\|x_{n+1}-x_{n}\right\| \\
& +\left\langle(\varsigma V-\mu D) x^{*}, x_{n}-x^{*}\right\rangle .
\end{aligned}
$$

It follows from (81) and (95) that

$$
\limsup _{n \rightarrow \infty}\left\langle(\varsigma V-\mu D) x^{*}, x_{n+1}-x^{*}\right\rangle \leq 0 .
$$


Observe that

$$
\begin{aligned}
\| x_{n+1} & -x^{*} \|^{2} \\
= & \left\|\alpha_{n} \varsigma V u_{n}+\left(I-\mu \alpha_{n} D\right) S_{n} u_{n}-x^{*}\right\|^{2} \\
= & \|\left(I-\mu \alpha_{n} D\right) S_{n} u_{n}-\left(I-\mu \alpha_{n} D\right) x^{*} \\
& +\alpha_{n}\left(\varsigma V u_{n}-\mu D x^{*}\right) \|^{2} \\
\leq & \left\|\left(I-\mu \alpha_{n} D\right) S_{n} u_{n}-\left(I-\mu \alpha_{n} D\right) x^{*}\right\|^{2} \\
& +2 \alpha_{n}\left\langle\varsigma V u_{n}-\mu D x^{*}, x_{n+1}-x^{*}\right\rangle \\
\leq & \left(1-\alpha_{n} \tau\right)^{2}\left\|u_{n}-x^{*}\right\|^{2} \\
& +2 \alpha_{n}\left\langle\varsigma V u_{n}-\gamma V x^{*}, x_{n+1}-x^{*}\right\rangle \\
& +2 \alpha_{n}\left\langle(\varsigma V-\mu D) x^{*}, x_{n+1}-x^{*}\right\rangle \\
\leq & \left(1-\alpha_{n} \tau\right)^{2}\left\|x_{n}-x^{*}\right\|^{2} \\
& +\alpha_{n} \varsigma l\left(\left\|x_{n}-x^{*}\right\|^{2}+\left\|x_{n+1}-x^{*}\right\|^{2}\right) \\
& +2 \alpha_{n}\left\langle(\varsigma V-\mu D) x^{*}, x_{n+1}-x^{*}\right\rangle .
\end{aligned}
$$

This implies that

$$
\begin{aligned}
\| x_{n+1} & -x^{*} \|^{2} \\
\leq & \frac{\left(1-\alpha_{n} \tau\right)^{2}+\alpha_{n} \varsigma l}{1-\alpha_{n} \varsigma l}\left\|x_{n}-x^{*}\right\|^{2} \\
& +\frac{2 \alpha_{n}}{1-\alpha_{n} \varsigma l}\left\langle(\varsigma V-\mu D) x^{*}, x_{n+1}-x^{*}\right\rangle \\
\leq & \left(1-\frac{2 \alpha_{n}(\tau-\varsigma l)}{1-\alpha_{n} \varsigma l}\right)\left\|x_{n}-x^{*}\right\|^{2} \\
& +\frac{2 \alpha_{n}}{1-\alpha_{n} \varsigma l}\left\langle(\varsigma V-\mu D) x^{*}, x_{n+1}-x^{*}\right\rangle+\frac{\left(\alpha_{n} \tau\right)^{2}}{1-\alpha_{n} \varsigma l} M_{3} \\
= & \left(1-\varphi_{n}\right)\left\|x_{n}-x^{*}\right\|^{2} \\
& +\frac{2 \alpha_{n}}{1-\alpha_{n} \varsigma l}\left\langle(\varsigma V-\mu D) x^{*}, x_{n+1}-x^{*}\right\rangle+\frac{\left(\alpha_{n} \tau\right)^{2}}{1-\alpha_{n} \varsigma l} M_{3},
\end{aligned}
$$

where $M_{3}=\sup _{n}\left\|x_{n}-x^{*}\right\|^{2}, n \geq 1$. It is easy to see that $\varphi_{n}=$ $2 \alpha_{n}(\tau-\varsigma l) /\left(1-\alpha_{n} \varsigma l\right)$. Hence by Lemma 2 , the sequence $\left\{x_{n}\right\}$ converges strongly to $x^{*}$.

Setting $V u_{n}=u$ in (78) in Theorem 11, we have the following result.

Corollary 12. Let $H_{1}$ and $H_{2}$ be two real Hilbert spaces and let $A: H_{1} \rightarrow H_{1}$ be a bounded linear operator, $f: C \rightarrow \mathbb{R} a$ real-value convex function, and $\nabla f$ an L-Lipschitzian mapping with $L \geq 0$. Let $u \in C$ be a fixed point in $C$. Assume that $\Omega:=$ $U \cap \Gamma \neq \emptyset$. Let $D$ be a strongly positive bounded linear operator with coefficients $\bar{\varsigma} \geq 0,0<\mu<2 \bar{\varsigma} /\|D\|^{2}$, and $0<\varsigma<(\mu(\bar{\varsigma}-$ $\left.\left.\left(\mu\|D\|^{2} / 2\right)\right) / l\right)=\tau / l$. Given $u, x_{0} \in H_{1}$ arbitrarily, let $\left\{x_{n}\right\}$ and $\left\{u_{n}\right\}$ be a sequence generated by the following algorithm:

$$
\begin{gathered}
u_{n}=J_{\lambda}^{B_{1}}\left(x_{n}+\xi A^{*}\left(J_{\lambda}^{B_{2}}-I\right) A x_{n}\right) ; \\
x_{n+1}=\alpha_{n} \varsigma u+\left(I-\mu \alpha_{n} D\right) S_{n} u_{n},
\end{gathered}
$$

where $\xi \in(0,1 / L), P_{C}\left(I-\gamma_{n} \nabla f\right)=\beta_{n} I+\left(1-\beta_{n}\right) S_{n}, S_{n}$ is nonexpansive, $\beta_{n}=\left(2-\gamma_{n} L\right) / 4, \gamma_{n} \in(0,2 / L)$, and $A^{*}$ is the adjoint of $A$ and $\left\{\alpha_{n}\right\},\left\{\beta_{n}\right\}$ satisfying conditions (i)-(ii) in Theorem 11 Then, the sequence $\left\{x_{n}\right\}$ converges strongly to a point $x^{*} \in \Omega$, which solves the variational inequality (40).

\section{Conflict of Interests}

The authors declare that there is no conflict of interests regarding the publication of this paper.

\section{Acknowledgments}

The authors thank the referees for comments and suggestions on this paper. The first author was supported by the Thailand Research Fund through the Royal Golden Jubilee Ph.D. Program (Grant no. PHD/0033/2554) and the King Mongkut's University of Technology Thonburi (KMUTT). Moreover, the second author was supported by the Thailand Research Fund and the King Mongkut's University of Technology Thonburi (Grant no. RSA5780059).

\section{References}

[1] Y. Censor and T. Elfving, "A multiprojection algorithm using Bregman projections in a product space," Numerical Algorithms, vol. 8, no. 2-4, pp. 221-239, 1994.

[2] C. Byrne, "Iterative oblique projection onto convex sets and the split feasibility problem," Inverse Problems, vol. 18, no. 2, pp. 441453, 2002.

[3] Y. Censor, T. Bortfeld, B. Martin, and A. Trofimov, "A unified approach for inversion problems in intensity-modulated radiation therapy," Physics in Medicine and Biology, vol. 51, no. 10, pp. 2353-2365, 2006.

[4] B. Eicke, "Iteration methods for convexly constrained ill-posed problems in Hilbert space," Numerical Functional Analysis and Optimization, vol. 13, no. 5-6, pp. 413-429, 1992.

[5] H.-K. Xu, "Averaged mappings and the gradient-projection algorithm," Journal of Optimization Theory and Applications, vol. 150, no. 2, pp. 360-378, 2011.

[6] M. Su and H. Xu, "Remarks on the gradient-projection algorithm," Journal of Nonlinear Analysis and Optimization, vol. 1, no. 1, pp. 35-43, 2010.

[7] H. K. Xu, "An iterative approach to quadratic optimization," Journal of Optimization Theory and Applications, vol. 116, no. 3, pp. 659-678, 2003.

[8] J.-L. Lions, Quelques Methodes de Resolution des Problemes aux Limites non Lineaires, Dunod, Paris, France, 1969.

[9] I. Yamada, “The hybrid steepest-descent mathod for variational inequality problems over the intersection of the fixed-point sets of nonexpansive mappings," in Inherently Parallel Algorithms 
in Feasibility and Optimization and Their Applications, D. Butnariu, Y. Censor, and S. Reich, Eds., pp. 473-504, NorthHolland, Amsterdam, The Netherlands, 2001.

[10] M. Tian, "A general iterative algorithm for nonexpansive mappings in Hilbert spaces," Nonlinear Analysis: Theory, Methods \& Applications, vol. 73, no. 3, pp. 689-694, 2010.

[11] A. Moudafi, "Split monotone variational inclusions," Journal of Optimization Theory and Applications, vol. 150, no. 2, pp. 275283, 2011.

[12] Y. Censor, A. Gibali, and S. Reich, "Algorithms for the split variational inequality problem," Numerical Algorithms, vol. 59, no. 2, pp. 301-323, 2012.

[13] P. L. Combettes, "The convex feasible problem in image recovery," in Advanced in Image and Electronphysics, P. Hawkes, Ed., vol. 95, pp. 155-270, Academic Press, New York, NY, USA, 1996.

[14] C. Byrne, Y. Censor, A. Gibali, and S. Reich, "The split common null point problem," Journal of Nonlinear and Convex Analysis, vol. 13, no. 4, pp. 759-775, 2012.

[15] K. R. Kazmi and S. H. Rizvi, "An iterative method for split variational inclusion problem and fixed point problem for a nonexpansive mapping," Optimization Letters, vol. 8, no. 3, pp. 1113-1124, 2014.

[16] T. Suzuki, "Strong convergence theorems for infinite families of nonexpansive mappings in general Banach spaces," Fixed Point Theory and Applications, vol. 2005, no. 1, Article ID 685918, pp. 103-123, 2005.

[17] K. Shimoji and W. Takahashi, "Strong convergence to common fixed points of infinite nonexpansive mappings and applications," Taiwanese Journal of Mathematics, vol. 5, no. 2, pp. 387404, 2001.

[18] S. Wang, "A general iterative method for obtaining an infinite family of strictly pseudo-contractive mappings in Hilbert spaces," Applied Mathematics Letters of Rapid Publication, vol. 24, no. 6, pp. 901-907, 2011.

[19] P. Duan, "General iterative methods for system of equilibrium problems and constrained convex minimization problem in Hilbert spaces," Journal of Applied Mathematics, vol. 2013, Article ID 957363, 11 pages, 2013.

[20] C. Byrne, "A unified treatment of some iterative algorithms in signal processing and image reconstruction," Inverse Problems, vol. 20, no. 1, pp. 103-120, 2004.

[21] P. L. Combettes, "Solving monotone inclusions via compositions of nonexpansive averaged operators," Optimization, vol. 53, no. 5-6, pp. 475-504, 2004. 


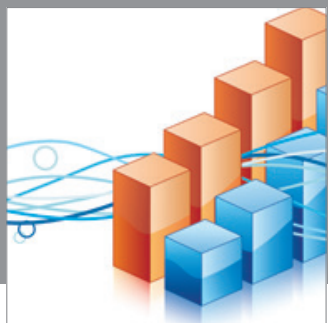

Advances in

Operations Research

mansans

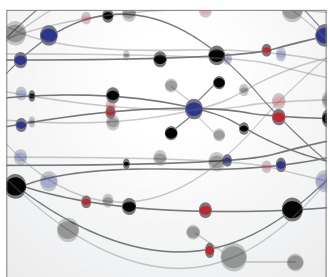

The Scientific World Journal
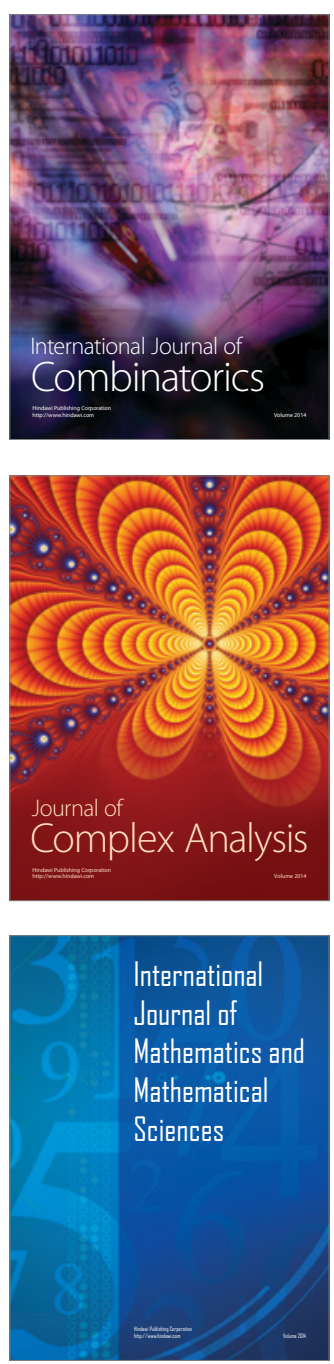
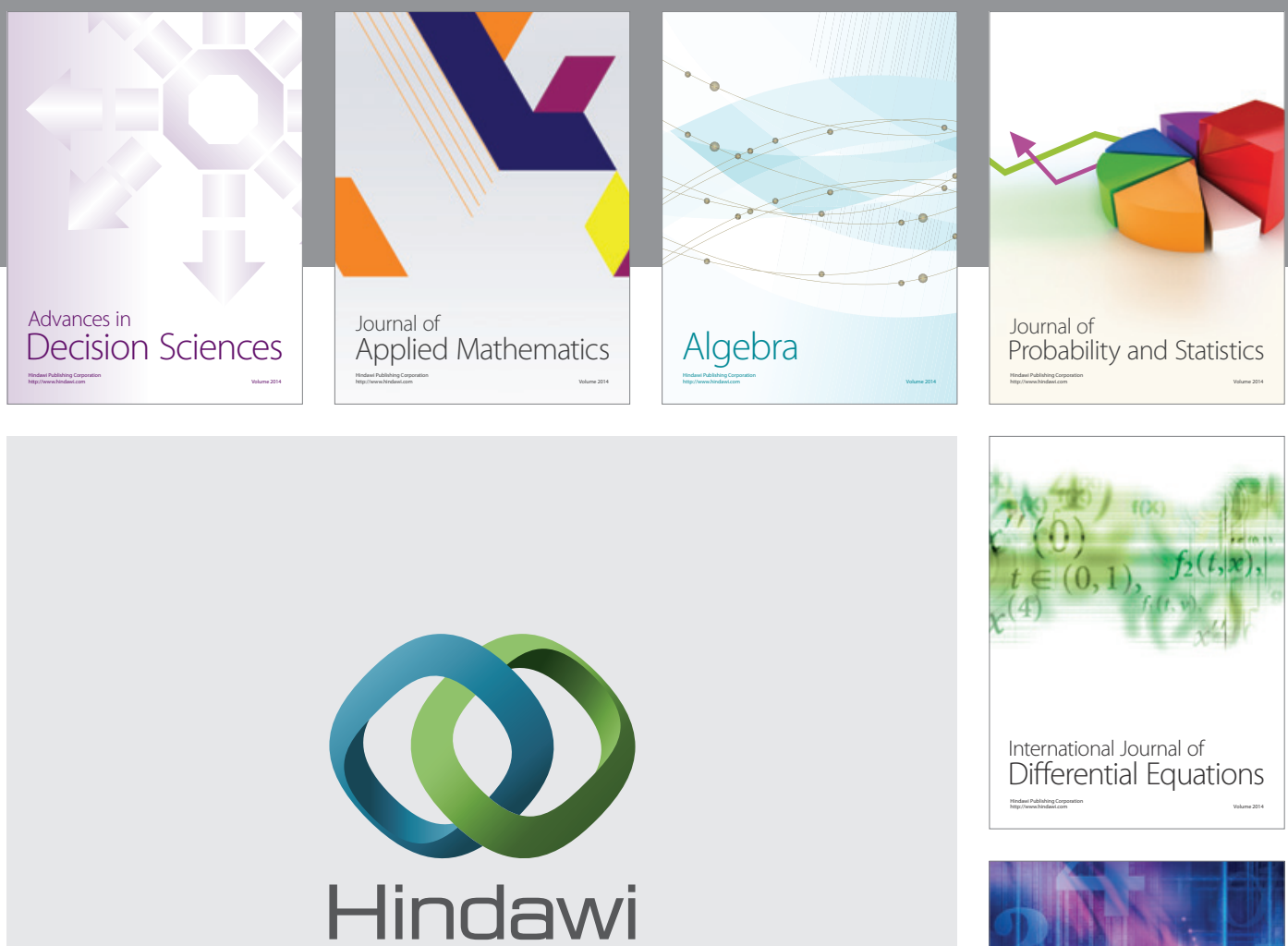

Submit your manuscripts at http://www.hindawi.com
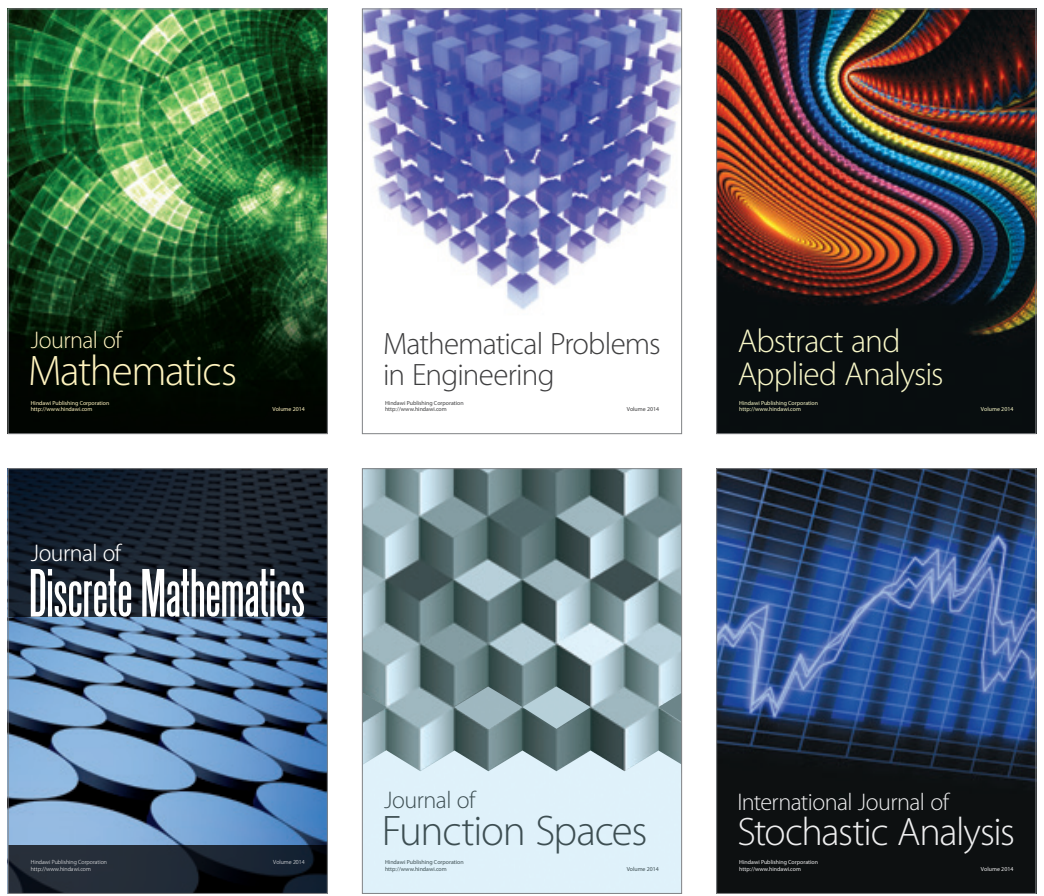

Journal of

Function Spaces

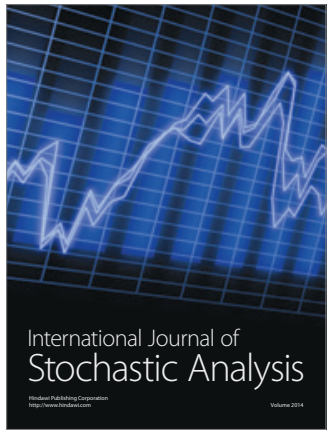

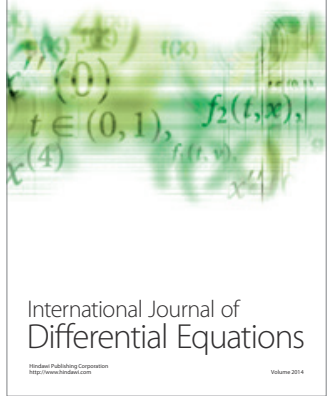
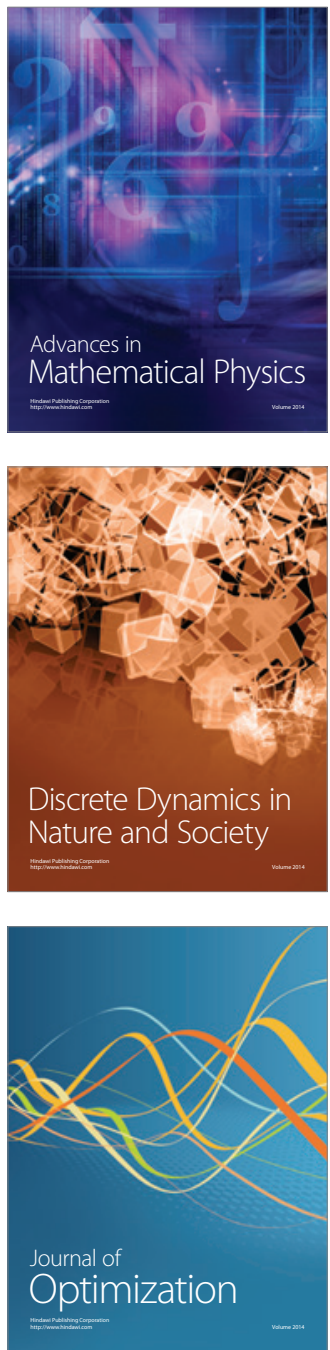